\title{
Effects of the parallel acceleration on heavy impurity transport in turbulent tokamak plasmas
}

\author{
Madalina Vlad, Dragos Iustin Palade, Florin Spineanu \\ National Institute of Laser, Plasma and Radiation Physics, Bucharest, Romania
}

August 26, 2020

\begin{abstract}
A process specific to the dynamics of the heavy impurities in turbulent tokamak plasmas is found and analysed. We show that the parallel stochastic acceleration is strongly coupled to the perpendicular transport and generates a radial pinch velocity. The interaction is produced with the hidden drifts, a quasi-coherent component of the motion that consists of a pair of average radial velocities in opposite directions. The parallel acceleration breaks this symmetry and yields a radial average velocity that can be in the inward or outward direction. The transport of the tungsten ions in three-dimensional turbulence is analysed in the frame of a test particle model using numerical simulations. The results show that the acceleration induced pinch can be important for $\mathrm{W}$ impurity transport in present days tokamaks and in ITER.
\end{abstract}

\section{Introduction}

Tungsten (W) will be used for plasma facing components in ITER, because this material fulfils essential requirements such as low erosion rate, low tritium retention and good thermal properties [1. The effects of the $\mathrm{W}$ wall on tokamak plasma performances have been intensively studied during the last decades in several tokamak devices (ASDEX Upgrade [2]-[4], JET [5]7], WEST [8, T-10 9], JT-60U [10]). The main drawback is the large radiation emission of these high charge ions that can strongly affect the energy balance if they accumulate in the core plasma in concentrations higher that $10^{-5}-10^{-4}$. It is, therefore, vital to acquire a good understanding of impurity transport and to develop methods for controlling the concentration of $\mathrm{W}$ in tokamak core plasmas. A large number of experimental [11]-[15], theoretical [16]-[20] and numerical [21]-27] studies have provided important results on these complex processes, but the domain is still open as shown by very recent papers [28]-[30]

The dynamics of the $\mathrm{W}$ ions includes both neoclassical and turbulent effects. The large mass and charge determine strong inertial and electrostatic forces, with the result of specific phenomena that are not observed at light ions. Strong poloidal asymmetries, significant increase 
of the neoclassical transport and accumulation on the low field side of the plasma or even around the magnetic axis characterize $\mathrm{W}$ ions. Radial convections with neoclassical or turbulence origins can determine $\mathrm{W}$ accumulation or decay.

The present paper deals with the turbulent transport of the heavy impurities. We analyze the effects of the parallel acceleration $a_{z}$ on the diffusion coefficients and on the radial pinch velocity. The acceleration scales as $a_{z} \sim Z / A$, where $Z$ is the ionization rate and $A$ is the mass number of the ions. Thus, it is smaller for the $\mathrm{W}$ impurities than for plasma ions. The factor $Z / A$ varies in the interval $(0.05,0.33)$ for the $W$ impurities, while it has the value 0.5 for the deuterium.

Surprisingly, we have found that the effect of $a_{z}$ can be significant for $W$ impurities, while it is negligible for plasma ions.

The main effect of the parallel acceleration consists of the generation of a radial pinch velocity $V_{x}$. The aim of the paper is to understand and to characterize this new mechanism of generating radial pinch.

The parallel acceleration is expected to influence impurity transport through the modification of the parallel decorrelation time. We show that, beside this direct effect, a much stronger coupling of the parallel accelerated motion to the radial transport appears. It consists of the perturbation of hidden drifts (HDs). The HDs are a pair of opposite velocities in the radial direction that appear in the presence of a poloidal average velocity [31]. This quasi-coherent motion has zero average and does not determine a convective velocity in the case of the ExB drift. The stochastic parallel acceleration perturbs the equilibrium of the HDs leading to a radial pinch. We show that this pinch mechanism can be relevant for $\mathrm{W}$ impurity accumulation in present days and in ITER plasmas.

The analysis is performed in the frame of a test particle stochastic model, which is shown to be the minimal model that yields this process. The model is presented in Section 2.

We use two theoretical methods for determining the pinch velocity and the transport coefficients, the direct numerical simulations (DNS) 32] and the decorrelation trajectory method (DTM) [33]. A three-dimensional DNS code for ion trajectories and for the calculation of the statistical Lagragian quantities was developed. It is described in Section 3.1. The DTM is a semi-analytical approach that provides approximate evaluations of the transport characteristics. It is presented in Section 3.2. The DTM is used for identifying and understanding qualitatively the new pinch mechanism, while the quantitative properties of the radial velocity $V_{x}$ are determined using the much more accurate results provided by DNS.

The effects of the parallel acceleration on the heavy impurity transport are identified in Section 4 by comparing typical results of the transport model with those obtained for deuterium ions and for $\mathrm{W}$ ions in two-dimensional potentials.

The physical processes that determine the generation of the radial pinch are discussed in Section 5. We use the DTM, which has the capability to provide physical pictures of complex nonlinear transport processes [34]-[36]. We show that the acceleration can have a strong influence on the HDs that essentially consists of the attenuation of one of the HDs, which compensates only partially the other HD yielding an average velocity. The physical image of the pinch generation mechanism is validated using DNS. A short discussion on the accuracy of the DTM is also presented in this Section. 
The properties of the pinch velocity $V_{x}$ and its dependence on the main parameters of the model are determined in Section 6. They are obtained using the more accurate results of the DNS. The study is focused on the scaling of $V_{x}$ with the main parameters of the model. The results are analyzed and physical explanations are derived.

The relevance of the pinch generated by the parallel acceleration for the $\mathrm{W}$ ion transport in the existing plasmas (ASDEX Upgrade and JET) and in ITER is discussed in Section 7. A summary of the results and the conclusions of this study are also included in this section.

\section{The transport model}

We study impurity transport in the slab approximation, at the low field side of the plasma. The magnetic field is constant along $\mathbf{e}_{z}$ axis and $\mathbf{x}=(x, y)$ is in the perpendicular plane, with $x$ the radial and $y$ the poloidal coordinate. The equations for the impurity ion trajectories are

$$
\begin{gathered}
\frac{d \mathbf{x}}{d t}=-\frac{\boldsymbol{\nabla} \phi \times \mathbf{e}_{z}}{B}+\mathbf{V}_{d}, \\
\frac{d z}{d t}=v_{z}, \quad \frac{d v_{z}}{d t}=-\frac{q}{m} \partial_{z} \phi,
\end{gathered}
$$

where the first term in Eq. (11) is the stochastic drift determined by the electric field of the turbulence $-\boldsymbol{\nabla} \phi(\mathbf{x}, z, t)(\phi(\mathbf{x}, z, t)$ is the stochastic potential, $\boldsymbol{\nabla}$ is the gradient in the perpendicular plane) and the second term $\mathbf{V}_{d}=V_{d} \mathbf{e}_{y}$ is a poloidal average velocity that can be produced by the magnetic drifts or plasma rotation. The parallel motion (2) includes the variation of the velocity determined by the stochastic acceleration $a_{z}=-q / m \partial_{z} \phi$.

The potential $\phi(\mathbf{x}, z, t)$ is modelled as a Gaussian random field with the Eulerian correlation (EC)

$$
E(\mathbf{x}, z, t) \equiv\langle\phi(\mathbf{0}, 0,0) \phi(\mathbf{x}, z, t)\rangle
$$

corresponding to drift type turbulence [34]-36]

$$
E(\mathbf{x}, z, t)=A_{\phi}^{2} \partial_{y}\left[\exp \left(-\frac{x^{2}}{2 \lambda_{x}^{2}}-\frac{y^{2}}{2 \lambda_{y}^{2}}-\frac{z^{2}}{2 \lambda_{z}^{2}}\right) \frac{\sin \left(k_{0} y\right)}{k_{0}}\right] T(t),
$$

where $A_{\phi}$ is the amplitude of the potential fluctuations, $\lambda_{x}, \lambda_{y}, \lambda_{z}$ are the correlation lengths along the radial, poloidal and parallel directions, and $k_{0}$ is the dominant wave number. The function $T(t)$ is the time correlation of the potential that is a decaying function of time with $\tau_{d}$ the decorrelation time

$$
T(t)=\exp \left(-\frac{t^{2}}{4 \tau_{d}^{2}}\right)
$$

Dimensionless quantities are used, with the units: $\rho_{i}=v_{t h i} / \Omega_{i}$, the Larmor radius of the protons (for the perpendicular distances, for the correlation lengths $\lambda_{x}, \lambda_{y}$ and for $1 / k_{0}$ ), $a$, the small radius of the plasma (for the parallel distances and for the correlation length $\lambda_{z}$ ), $\tau_{0}=a / v_{t h i}$ (for time and for $\tau_{d}$ ), $A_{\phi}$ (for the potential $\phi$ ), $V_{*}=\rho_{i} v_{t h i} / a$ (for the perpendicular velocities and $V_{d}$ ) and $v_{t h W}=v_{t h i} / \sqrt{A}$ (for the parallel velocity of the $\mathrm{W}$ ions). $v_{t h i}=\sqrt{T_{i} / m_{p}}$ 
is the thermal velocity of protons with temperature $T_{i}$ and mass $m_{p}$ and $\Omega_{i}=e B / m_{p}$ is the cyclotron frequency of the protons. The notations are not changed for the dimensionless quantities, and Eqs. (11)-(2) for ions with mass number $A$ and ionization rate $Z$ are

$$
\begin{aligned}
& \frac{d x}{d t}=-K_{*} \partial_{y} \phi(\mathbf{x}, z, t), \frac{d y}{d t}=K_{*} \partial_{x} \phi(\mathbf{x}, z, t)+V_{p}, \\
& \frac{d z}{d t}=\frac{1}{\sqrt{A}} v_{z}, \quad \frac{d v_{z}}{d t}=-P_{a} \partial_{z} \phi .
\end{aligned}
$$

The main characteristics of the model appear in three dimensionless parameters evidenced in the dimensionless equation. The parameter $K_{*}$ is the dimensionless measure of turbulence amplitude

$$
K_{*}=\Phi \frac{a}{\rho_{i}}, \quad \Phi=\frac{e A_{\phi}}{T_{i}} .
$$

The parameter of the poloidal velocity $V_{p}$ is

$$
V_{p} \equiv \frac{V_{d}}{V_{*}}=\frac{V_{d}}{v_{t h i}} \frac{a}{\rho_{i}}
$$

The parameter of the parallel acceleration $a_{z}$ is

$$
P_{a} \equiv \Phi \frac{Z}{\sqrt{A}} .
$$

We note that the first two parameters that describe the perpendicular motion depend on plasma size factor $\rho_{*}=\rho_{i} / a$.

The energy of the ions normalized with the temperature $T_{i}$ is

$$
W=\frac{1}{2} v_{z}^{2}+Z \Phi \phi
$$

It is the invariant of the motion in three-dimensional static potentials $\phi(\mathbf{x}, z)$. This constraint influences the transport for $\tau_{d} \rightarrow \infty$, and its effects persist in the case of potentials with slow time variation (large $\tau_{d}$ ). We note that the energy is dominated by the potential energy for the $\mathrm{W}$ ions with large $Z$, even at small turbulence amplitudes $\left(\Phi \sim 10^{-2}\right)$.

\section{Theoretical methods}

The model is analysed using direct numerical simulations (DNS) 32] and the decorrelation trajectory method (DTM) [33].

\subsection{DNS numerical methods and code}

The numerical methods used in the DNS code are described and analyzed in [32. A series of fast numerical generators of Gaussian random fields with given EC are proposed. In the present work, we have implemented the so called FRD representation

$$
\phi(\mathbf{X})=\sum_{i=1}^{N_{c}} \sqrt{S\left(\mathbf{K}_{i}\right)} \sin \left(\mathbf{K}_{i} \mathbf{X}+\frac{\pi}{4} \zeta_{i}\right),
$$


where $\mathbf{X} \equiv(\mathbf{x}, z, t)$ is the four-dimensional space-time, $\mathbf{K}_{i} \equiv\left(\mathbf{k}_{\perp}, k_{z}, \omega\right)$ are the $N_{c}$ discrete values of the corresponding wave numbers and frequency and $S(\mathbf{K})$ is the spectrum of the stochastic potential (the Fourier transform of the EC (44)). This representation is different of the usual discrete Fourier decomposition by the set of the values of $\mathbf{K}_{i}$ that are not the fixed points of a four-dimensional mesh, but random values with uniform distribution. Also, the random phases do not have continuous distributions, but discrete values \pm 1 (with equal probabilities). Each set of the $N_{c}$ random values $\zeta_{i}$ determines a realization of the potential, which constitutes the statistical ensemble (with a number $M$ of elements).

We have shown 32 that the representation (12) provides fast convergence of the Eulerian statistics of the generated fields, as well as of the Lagrangian statistics of trajectories. In particular, it was proven that a convergence level with a few percents error can be achieved with $N_{c} \sim 10^{d}$ and $M \sim 10^{4}$, where $d=4$ for time dependent potentials and $d=3$ for $\tau_{d} \rightarrow \infty$. Also, it is worth mentioning that such representations are able to reproduce with high accuracy the conservation laws of motion as well as certain Lagrangian statistical invariants.

The properties of the representation (12) enables to use commonly in the present simulations $N_{c} \sim 1-5 \times 10^{3}$ partial waves. The dimension of the statistical ensemble is usually set to $M \sim 10^{5}$ realizations which gives negligible statistical fluctuations. The numerical integration scheme used is a forth order Runge-Kutta method which preserves well the energy with a minimal numerical effort. Depending on the integration time and on the type of turbulence (frozen, or not), the usual CPU times on personal computer are $t_{C P U} \sim 2-20$ hours per run.

\subsection{DTM semi-analytical method}

The DTM is a semi-analytical approach, which is able to describe both the random and the quasi-coherent components of the trajectories. The latter are determined by the finite correlation lengths of the stochastic potential and depend on the structure of the correlated zone that is described by the shape of the EC.

The statistical ensemble of stochastic potentials is divided in subensembles $S$ with given values of the potential and of its derivatives at the origin of the trajectories, $\mathbf{x}=\mathbf{0}, z=0, t=0$

$$
\phi(\mathbf{0}, 0,0)=\phi^{0}, \partial_{i} \phi(\mathbf{0}, 0,0)=\phi_{i}^{0},
$$

where $i=x, y, z$. The potential and its derivatives, restricted at the realizations contained in a subensemble, are Gaussian fields with space-dependent averages

$$
\begin{gathered}
\langle\phi(\mathbf{x}, z, t)\rangle_{S} \equiv \Phi^{S}(\mathbf{x}, z, t)=\phi^{0} \frac{E(\mathbf{x}, z, t)}{E(\mathbf{0}, 0,0)}-\sum_{i} \phi_{i}^{0} \frac{E_{i}(\mathbf{x}, z, t)}{E_{i i}(\mathbf{0}, 0,0)} \\
\left\langle\partial_{i} \phi(\mathbf{x}, z, t)\right\rangle_{S}=\partial_{i} \Phi^{S}(\mathbf{x}, z, t)
\end{gathered}
$$

where $E_{i j}$ are derivatives of the EC, $E_{i}=\partial_{i} E, E_{i i}=\partial_{i}^{2} E$. The amplitudes of fluctuations in a subensemble vanishes in $\mathbf{x}=\mathbf{0}, z=0$, and they reach the level corresponding to the whole set of realizations only at large distances compared to the correlation lengths.

Particle trajectories are studied separately in each subensemble $S$. The average potential (14), determined by the EC, yields an average trajectory in each subensemble. It is obtained 
by averaging Eqs. (6), (7) over the realizations that belong to $S$. Neglecting the fluctuations of the potential in $S$ (see [33, [37] for the discussion of this approximation), one obtains a system of subensemble average equations (S-eq). It has the same structure as Eqs. (6), (77), but with the stochastic potential $\phi(\mathbf{x}, z, t)$ replaced by the subensemble average potential $\Phi^{S}(\mathbf{x}, z, t)$. The solution of the (S-eq), $\mathbf{X}\left(t ; \phi^{0}, \phi_{i}^{0}\right), Z\left(t ; \phi^{0}, \phi_{i}^{0}\right)$, is a smooth, simple trajectory, which is named decorrelation trajectory (DT) because it represents the average evolution of the particles through the correlated zone of the potential. An important feature of the DTs is that they obey any conservation law which characterize the real trajectories. In our particular case, the energy

$$
\langle W(t)\rangle_{S}=\frac{1}{2}\left\langle v_{z}^{2}(t)\right\rangle_{S}+Z \Phi \Phi^{S}\left(\mathbf{X}\left(t ; \phi^{0}, \phi_{i}^{0}\right), Z\left(t ; \phi^{0}, \phi_{i}^{0}\right)\right)
$$

is conserved along the DTs for static potentials.

The statistical characteristics of the stochastic trajectories are obtained as weighted averages along the DTs by summing the contributions of all subensembles. In particular, the time dependent diffusion coefficient and the average radial displacement are

$$
\begin{aligned}
D_{x}(t) & =\int d \phi^{0} d \phi_{x}^{0} d \phi_{y}^{0} d \phi_{z}^{0} P\left(\phi^{0}, \phi_{i}^{0}\right) v_{x}^{0} X\left(t ; \phi^{0}, \phi_{i}^{0}\right), \\
X(t) & \equiv\langle x(t)\rangle=\int d \phi^{0} d \phi_{x}^{0} d \phi_{y}^{0} d \phi_{z}^{0} P\left(\phi^{0}, \phi_{i}^{0}\right) X\left(t ; \phi^{0}, \phi_{i}^{0}\right),
\end{aligned}
$$

where $P\left(\phi^{0}, \phi_{i}^{0}\right)$ is the (Gaussian) probability of the initial conditions (13) and $v_{x}^{0}=-K_{*} \phi_{y}^{0}$ is the initial radial velocity.

The average displacement generates an average velocity for stochastic processes that have finite decorrelation times. The parallel motion provides an intrinsic decorrelation process that yields from the $z$-dependence of the EC

$$
\tau_{z}^{e f f}(t)=\int_{0}^{t} d \tau\left\langle\exp \left(-\frac{z^{2}(\tau)}{2 \lambda_{z}^{2}}\right)\right\rangle .
$$

This is an increasing function of time that saturates at a finite value $\tau_{z}^{\infty}$. The radial pinch velocity is evaluated as

$$
V_{x}(t)=\frac{\left\langle x\left(\tau_{z}^{e f f}(t)\right)\right\rangle}{\tau_{z}^{e f f}(t)}, \quad V_{x}^{\infty}=\frac{\left\langle x\left(\tau_{z}^{\infty}\right)\right\rangle}{\tau_{z}^{\infty}}
$$

We note that, in time dependent potentials, $\tau_{z}^{e f f}(t)$ combines with the time dependence of the EC $T(t)$ in a modified function $\tau^{e f f}(t)$ with a modified asymptotic value $\tau^{\infty}$.

The time dependent functions $D_{x}(t)$ (17) and $V_{x}(t)$ (20) provide details of the transport process, while their asymptotic values $D_{x}^{\infty}, V_{x}^{\infty}$ represent the diffusion coefficient and the pinch velocity that determine the impurity flux at the transport space-time scale. 


\section{Effects of the parallel acceleration}

Typical results obtained for $\mathrm{W}$ ions with $Z=40$ in a turbulent plasma with the parameters $\Phi=0.03, V_{d}=1, \lambda_{x}=5, \lambda_{y}=2, \lambda_{z}=1, k_{0}=1, \tau_{d}=\infty$ and $a / \rho_{i}=500$ are presented Figure 1. The time-dependent diffusion coefficient $D_{x}(t)$ and the radial average velocity $V_{x}(t)$ are shown (solid lines) compared to case of deuterium (D) ions (dashed-dotted lines) and to $\mathrm{W}$ ions with $a_{z}=0$ (dashed lines).
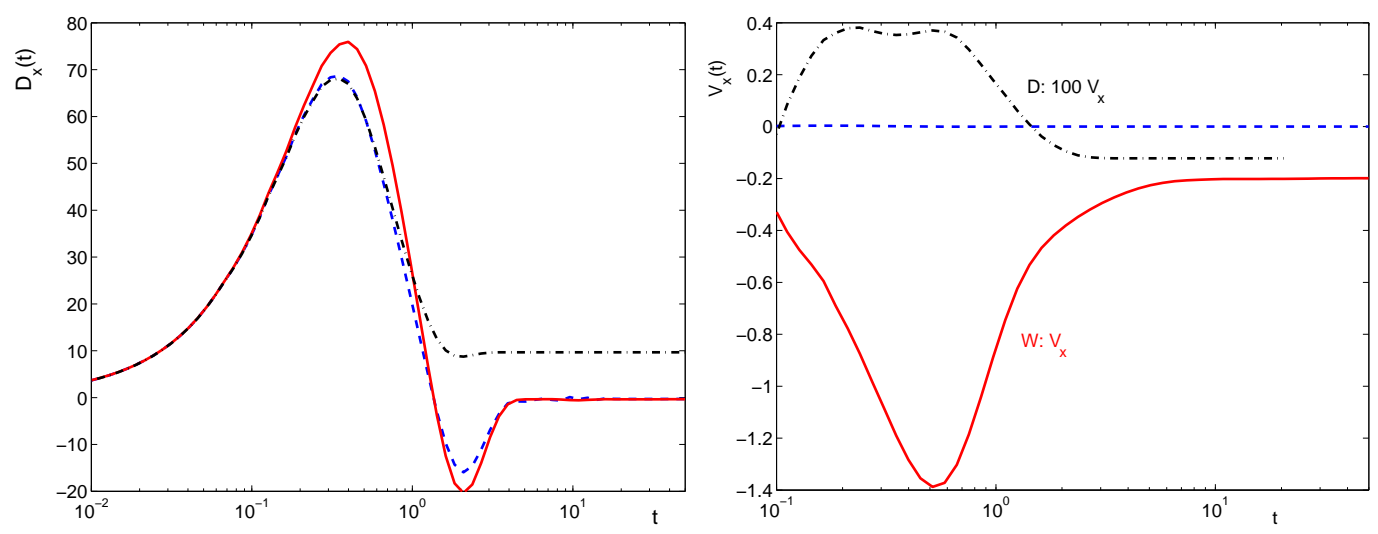

Figure 1: Typical results for $\mathrm{W}$ and $\mathrm{D}$ ions: the time dependent diffusion coefficient $D_{x}(t)$ (left panel) and the average radial velocity $V_{x}(t)$ (right panel). The continuous (red) lines are for $\mathrm{W}$ ions with parallel acceleration, the dashed (blue) lines are for the $\mathrm{W}$ ions with $a_{z}=0$ and the dashed-dotted (black) lines are for D ions with parallel acceleration.

The diffusion coefficients shown in Fig. 1 (left panel) have a similar time dependence for all three cases. The increase at small times corresponds to the quasilinear regime that is identical for all examples. It lasts for $t \ll \tau_{f l}$, where $\tau_{f l}$ is the time of flight defined as the ratio of $\lambda_{x}$ and the amplitude of the stochastic radial velocity, which in this units is $\tau_{f l}=\lambda_{x} \lambda_{y} / K_{*}$. The maximum of $D_{x}(t)$ appears at $\tau_{f l}=0.66$ and, at larger times, the eddying (trapping) determines the decay of $D_{x}(t)$ that lasts until the decorrelation of the trajectories from the potential produces the saturation. One can see that the saturation is at a much smaller time for the $\mathrm{D}$ ions than for the $\mathrm{W}$ ions, which means that the parallel decorrelation time $\tau_{z}^{\infty}$ is much smaller in the first case. The parallel acceleration does not change the result for the D ions. The same result is obtained with/without $a_{z}$ (the dashed-dotted line). The parallel acceleration determines a modification of $D_{x}(t)$ for the $\mathrm{W}$ ions (see the solid curve compared to the dashed one). It essentially consists, in this case, of a small increase of the time of flight.

The main effect of the parallel acceleration is the generation of a radial pinch. As seen in Fig. 1 (right panel), a negative (inward) average velocity $V_{x}(t)$ appears due to $a_{z}$. It has a transitory large increase until $t \sim \tau_{f l}$, then it decays and eventually saturates due to the parallel decorrelation. When $a_{z}$ is neglected, $V_{x}(t)=0$ at any time. The radial pinch is much smaller for $\mathrm{D}$ ions than for the $\mathrm{W}$ ions. As seen in the figure, only multiplied by 100 the $\mathrm{D}$ pinch velocity (dashed-dotted curve) reaches values comparable to the $\mathrm{W}$ pinch velocity (solid curve). 
These much larger effects of the parallel acceleration on the heavy impurity transport compared to the case of $\mathrm{D}$ ions are rather surprising, because the normalized parallel acceleration in Eq. (77) scales as $d v_{z} / d t \sim P_{a} \sim Z / \sqrt{A}$. It is smaller by a factor 0.3 for $\mathrm{W}$ compared to $\mathrm{D}$ ions, which strongly enables to predict very small effects on heavy impurity transport.

The interaction of the parallel motion with the parallel transport is the effect of the finite parallel correlation length $\lambda_{z}$, which makes the EC (3) a $z$-dependent function that decays with the increase of $z$. The average of the EC over the parallel motion $z(t)$, solution of Eq. (7), yields a time decaying function. This parallel decorrelation process has the characteristic time $\tau_{z}^{\infty}$, which is the asymptotic value of the effective parallel time defined in Eq. (19). The values of $\tau_{z}^{\infty}$ and its scaling with the parameters of the parallel motion are different for the $\mathrm{D}$ and $\mathrm{W}$ ions, as shown below.

The variation range of the parallel velocity results from the energy conservation

$$
v_{z}= \pm \sqrt{2(W-Z \Phi \phi)}
$$

while its dynamics, reflected in the variation time, is determined by the parallel acceleration $a_{z}$.

At $Z=1$, the potential energy is small, $\Phi \ll W$, and the velocity can be approximated by $v_{z} \cong \pm \sqrt{2 W}(1-\Phi / 2 W)$. The acceleration determines for the $\mathrm{D}$ ions only a small fluctuation of $v_{z}(t)$ around the effective values $v_{z}^{\text {eff }} \cong \pm \sqrt{2 W}$. The parallel displacements are $z= \pm$ $\sqrt{2 W / A} t$, and the decorrelation time is approximated by $\tau_{z}^{\infty} \cong \lambda_{z} / \sqrt{W}[38$.

Thus, the parallel decorrelation time is practically not modified by $a_{z}$ at small $Z$, because $v_{z}^{e f f}$ does not depend on $\Phi$ and $\lambda_{z}$.

At large $Z$, the potential energy is large, of the order of the total energy $W, Z \Phi \sim W$. In these conditions, the trajectories cannot reach the regions with large, positive $\phi$ and the Lagrangian potential has an upper limit

$$
\phi(\mathbf{x}(t), z(t))<\phi_{\max }=\frac{W}{Z \Phi} .
$$

In addition, the kinetic energy is larger than the total energy $W$ in the regions with negative potential. Both the average and the fluctuation amplitude of $v_{z}(t)$ are functions of $W, Z$ and $\Phi$. They also depend on $\lambda_{z}$ through the characteristic variation time of $v_{z}(t)$, which is determined by the acceleration $a_{z} \sim 1 / \lambda_{z}$. The analytical estimation of $v_{z}^{\text {eff }}\left(W, Z, \Phi, \lambda_{z}\right)$ and $\tau_{z}^{\infty}\left(W, Z, \Phi, \lambda_{z}\right)$ is not possible in this case, but only the general behaviour with the parameters of the parallel motion. The range of variation of $\left|v_{z}(t)\right|$ is the interval $[0, W+Z \Phi)$, where the lower limit is determined by $\phi_{\max }$ in Eq. (22) and the upper limit corresponds to the amplitude of the order $-\Phi$ of the negative potential. This shows that $v_{z}^{e f f}$ increases with $W, Z$ and $\Phi$, and that $v_{z}^{e f f}\left(W, Z, \Phi, \lambda_{z}\right)>\sqrt{2 W}$. The dynamics of $v_{z}(t)$ that is determined by $a_{z} \sim P_{a} / \lambda_{z}$ leads to the decrease of $v_{z}^{e f f}$ at the increase of $\lambda_{z}$.

Thus, the parallel decorrelation time is modified by $a_{z}$ at large $Z$, and depends on all the parameters of the parallel motion. It can be approximated by

$$
\tau_{z}^{\infty}\left(W, Z, \Phi, \lambda_{z}\right) \cong \lambda_{z} \sqrt{A} / v_{z}^{e f f}
$$

which is a decreasing function of $W, Z, \Phi$ and an increasing function of $\lambda_{z}\left(\right.$ as $\lambda_{z}^{\alpha}$ with $\left.\alpha>1\right)$. 
The influence of the decorrelation time on the diffusion is different in the quasilinear $\left(\tau_{z}^{\infty}<\right.$ $\left.\tau_{f l}\right)$ and the trapping $\left(\tau_{z}^{\infty}>\tau_{f l}\right)$ regimes. The asymptotic diffusion coefficient scales as

$$
D_{x}^{\infty} \sim \begin{cases}\Phi^{2} \tau_{z}^{\infty}, & \tau_{z}^{\infty}<\tau_{f l} \\ \Phi^{\gamma} \tau_{z}^{\gamma-1}, & \tau_{z}^{\infty}>\tau_{f l}\end{cases}
$$

where $0<\gamma<1$.

The effects of the interaction of the parallel motion with the perpendicular transport through the parallel decorrelation explain the results obtained for the diffusion coefficients. The influence of the parallel acceleration is negligible for the $\mathrm{D}$ ions and noticeable for $\mathrm{W}$ ions. The strongest difference appears due to the dependence of $\tau_{z}^{\infty}$ on the mass number, which leads for the case presented in Figure 1 (left panel) to $\tau_{z}^{\infty}=1.15$ for the D ions (dashed-dotted line) and $\tau_{z}^{\infty}=11.25$ for the $\mathrm{W}$ ions (dashed line). The diffusion is in the trapping regime in both cases, which corresponds, according to Eq. (24), to much smaller $D_{x}^{\infty}$ for the W ions compared to D ions, as seen in Figure 1 (left panel).

The the radial pinch velocity seen in Figure 1 (right panel) cannot be explained by the parallel decorrelation process. A different interaction process provides the physical mechanism of pinch generation, as demonstrated in the next section.

\section{The pinch mechanism}

Particle trajectories described by Eqs. (11)-(2) with $\lambda_{z} \rightarrow \infty$ (two-dimensional potentials) have both stochastic and quasi-coherent aspects. The coherent motion is determined by the trapping or eddying in the structure of the potential, which determine small structures that produce a micro-confinement process [37]. It hinders the diffusive transport by decreasing the diffusion coefficient. The quasi-coherent component of the motion can also yield flows [39]-[41]. We have shown [31] that a special quasi-coherent effect, that is neither structure nor flow, appears in the stochastic transport in the presence of an average poloidal velocity $V_{p}$. It consists of two average radial velocities in opposite directions, which exactly compensate. This pair of drifts are named in [31 hidden drifts (HDs) because they do not yield an average velocity in these conditions.

The HDs are essentially determined by the existence of average displacements of the trajectories that start from same values of the potential $\phi^{0}$, and by the special property of these conditional averages $\langle\mathbf{x}(t)\rangle_{\phi^{0}}$ of having the sign correlated to the sign of $\phi^{0}$. These quantities, evaluated by DTM from Eq. (18), are

$$
\langle x(t)\rangle_{\phi^{0}}=\int d \phi_{x}^{0} d \phi_{y}^{0} d \phi_{z}^{0} P\left(\phi^{0}, \phi_{i}^{0}\right) X\left(t ; \phi^{0}, \phi_{i}^{0}\right) .
$$

The conditional displacements are zero in the case of the motion determined only by the electric drift, but they have finite values in the presence of an average poloidal velocity $V_{p}$. In the absence of the parallel acceleration (two-dimensional potentials), $\langle\mathbf{x}(t)\rangle_{\phi^{0}}$ is an antisymmetrical function of $\phi^{0}$ and it leads, by integration over $\phi^{0}$, to zero average displacement. A typical example is shown in Fig. 2 (left panel), which corresponds to the transport of the W ions with $a_{z}=0$ represented in Fig. 1 by the dashed lines. 

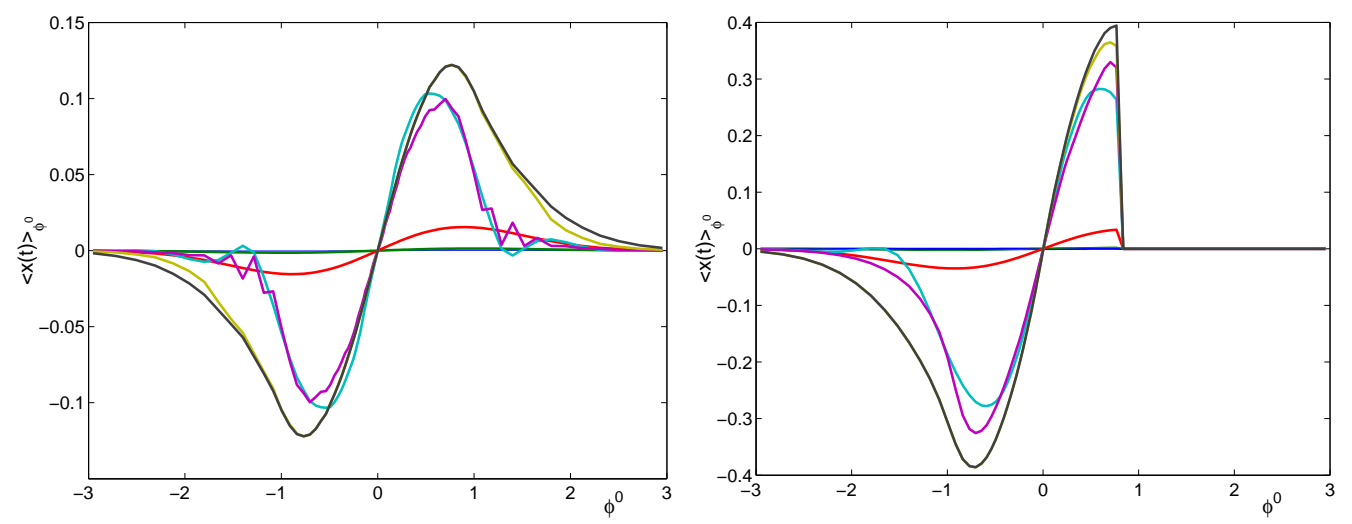

Figure 2: The average displacements conditioned by the initial potential $\phi^{0}$ as functions of $\phi^{0}$ for several times. The parallel acceleration is neglected in the left panel and is considered in the right panel.

This special type of quasi-coherent motion is generated by the average poloidal velocity $V_{p}$, which is equivalent with an average potential that adds to the stochastic potential. The contour lines of the total potential $\phi_{t}(\mathbf{x})=\phi(\mathbf{x})+x V_{p}$ show a complex structure having strips of open lines that oscillate between islands of closed lines. The trajectories are along the contour lines of the potential, and thus they are of two types: trapped (closed) and free (with unlimited displacements along $\mathbf{V}_{p}$ ). The probability of finding the trajectory along a closed contour line is larger on the side on which $\mathbf{V}_{p}$ is opposite to the stochastic velocity than on the other side. This leads to average radial displacements on the contour line $\phi^{0}$ that are positive for $\phi^{0}>0$ and negative for $\phi^{0}<0$. The free trajectories also contribute to the ordered conditional displacements. The Lagrangian invariance of $\phi_{t}(\mathbf{x}(t))=\phi(\mathbf{x}(t))+x(t) V_{p}=\phi^{0}$ constrains these trajectories to oscillate around the line $x=\phi^{0} / V_{p}$, which is the average for the trajectories that start from $\phi^{0}$.

An important property can be deduced from the physical image of the quasi-coherent process generated by the poloidal average velocity. The change of the sign of $V_{p}$ determines the change of the sign of the conditional average displacement $\langle\mathbf{x}(t)\rangle_{\phi^{0}}$.

The average displacements conditioned by the sign of the potential

$$
\langle x(t)\rangle_{+}=\int_{0}^{\infty} d \phi^{0}\langle x(t)\rangle_{\phi^{0}},\langle x(t)\rangle_{-}=\int_{-\infty}^{0} d \phi^{0}\langle x(t)\rangle_{\phi^{0}}
$$

determine, using Eq. (20), two opposite radial velocities $V_{+}, V_{-}$that exactly compensate $V_{+}+V_{-}=0$ due to the anti-symmetry of $\langle x(t)\rangle_{\phi^{0}}$ with respect to the initial potential $\phi^{0}$ (see [31, [42] for details).

The HDs represent a reservoir for direct transport, because perturbations produced by other components of the motion can affect the equilibrium of the HDs leading to an average velocity. We have shown [42] that the polarization drift determines a significant modification of the symmetry of the HDs and provides a mechanism for radial pinch generation. Essentially, this pinch appears due to the compressibility effect of the polarization drift. 
We show here that the three-dimensional stochastic motion described by Eqs. (11)-(2) can influence the equilibrium of the HDs.

An important difference between the three-dimensional and two-dimensional motion is that the Lagrangian potential is not invariant. This means that the trajectories in the subensemble $\mathrm{S}$ do not evolve on the contour lines $\phi(\mathbf{x})=\phi^{0}$, but they move up and down $\phi(\mathbf{x}, z)$ according to the parallel acceleration and velocity. As a consequence, the conditional averages $\langle x(t)\rangle_{\phi^{0}}$ undergo a complex averaging process that influences their anti-symmetrical dependence on $\phi^{0}$. The parallel acceleration moves the trajectories toward the minima of the stochastic potential, which favours the $\langle x(t)\rangle_{\phi^{0}}$ with $\phi^{0}<0$. On the other hand, the parallel velocity increases in the regions with negative potential and decreases in the regions with positive potential. The ions spend smaller time at negative than at positive potential, which partly compensates the attraction towards the potential minima, and yields a small perturbation of the symmetry of the HDs.

This explains the small pinch velocity observed for $\mathrm{D}$ ions.

The heavy ions with large ionization rates $Z$ have smaller acceleration, but also a much higher potential energy (larger than for $\mathrm{D}$ ions by the factor $Z$ ). Then, even for small amplitudes of the turbulence ( $\Phi$ of the order $10^{-2}$ ), the trajectories cannot reach the maxima of the stochastic potential, because the invariance of the energy (11) imposes $W-Z \Phi \phi^{0}=v_{z}^{2} / 2>0$. The result is the cut of $\langle x(t)\rangle_{\phi^{0}}$ at large $\phi^{0}$, as seen in Figure 2.b (obtained with DTM for the case presented by the solid lines in Figure 1). A strong symmetry breaking of the conditional displacements is produced at large $Z$, which yields, using Eq. (201), an average radial velocity $V_{x}(t)$. The maximum allowed potential $\phi_{\max }$ Eq. (22) decreases with the increase of $Z$, which leads to the increase of the average displacement and of the pinch velocity $V_{x}^{\infty}$.

This explains the significant pinch velocity produced by the parallel acceleration for $\mathrm{W}$ ions.

The physical mechanism for the generation of the radial pinch is validated using DNS. Fig. 3 confirms the existence of the conditional displacements $\langle x(t)\rangle_{\phi^{0}}$ and the perturbation produced by the parallel acceleration, which essentially consists of forbidding the trajectories to reach the maxima of the potential. It yields average displacements as function of the initial value of the potential that are similar to those obtained by DTM (shown in Fig. 2 (right panel)).

The time dependent pinch velocity and diffusion coefficient obtained from the numerical simulation of the stochastic trajectories are presented in Fig. 4. They correspond to the W ion transport for the set of parameters mentioned at the beginning of this Section, which yield using DTM the results shown in Fig. 1 (continuous lines). One can see that the results of the simulation are similar to those of the DTM for both $V_{x}(t)$ and $D_{x}(t)$. This shows that DTM is qualitatively adequate for the study of the three-dimensional model (1)-(2). This conclusion is in agreement with previous studies [43.

There are, however, important differences that result from the approximation used in the DTM. They consists of the overestimation of trajectory trapping, which leads to smaller $D_{x}^{\infty}$ and larger $V_{x}^{\infty}$. The overestimation of the pinch velocity is stronger in the case of time dependent potentials. The results presented in the next Section are obtained using DNS. 


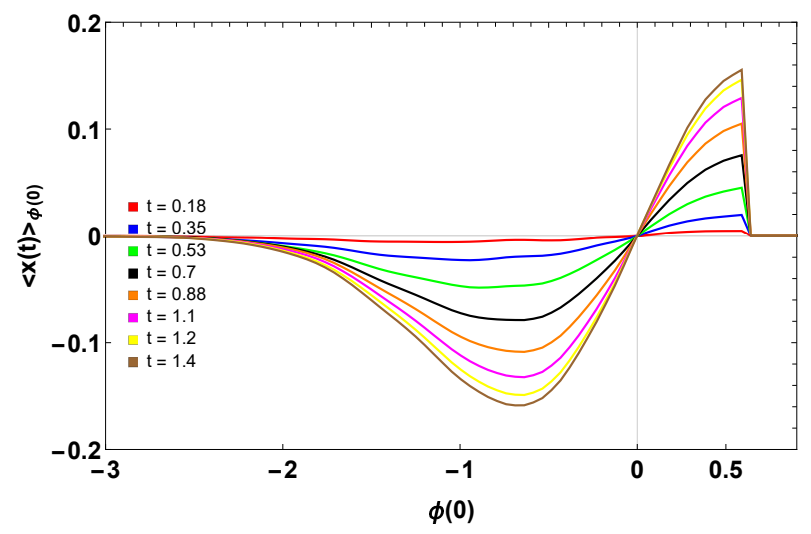

Figure 3: Validation of the pinch mechanism by DNS. The conditional displacements $\langle x(t)\rangle_{\phi^{0}}$ obtained with DNS.
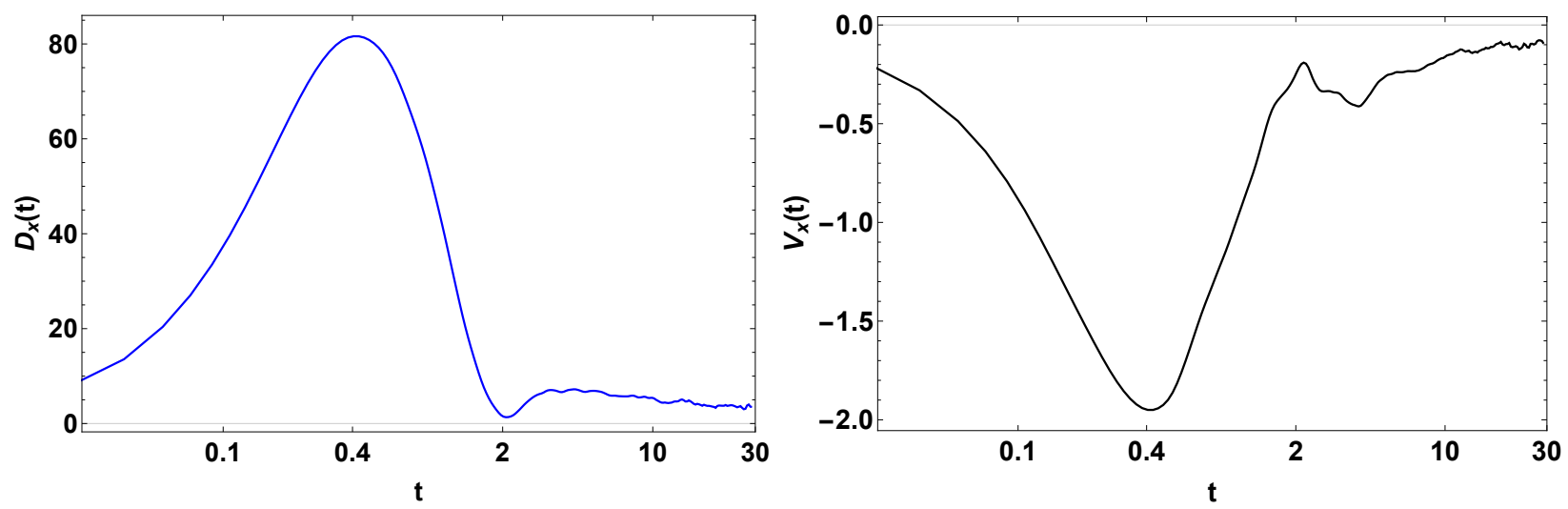

Figure 4: Validation of the pinch mechanism by DNS. The time dependent diffusion coefficient $D_{x}(t)$ (left panel) and the average radial velocity $V_{x}(t)$ (right panel).

\section{Characterization of the pinch velocity}

The impurity ion transport described by Eqs. (1)-(2) depends on a large number of parameters. Taking the case of W ions in large size plasmas of ITER type, we fix $A=184$ and $\rho_{*}=\rho_{i} / a=$ $1 / 500$, and remain with 9 dimensionless parameters: $K_{*}, \lambda_{z} / a, V_{p} / V_{*}, P_{a}, \tau_{d} / \tau_{0}, W, \lambda_{x} / \rho_{i}$, $\lambda_{y} / \rho_{i}$ and $k_{0} \rho_{i}$.

The first three parameters are essential because they describe the main ingredients of the pinch mechanism: a turbulent state of plasma $\left(K_{*}\right)$ with three-dimensional stochastic potential (finite $\lambda_{z}$ ) and an average poloidal velocity $\left(V_{p}\right)$. The characteristics of the impurity ions are represented by $P_{a}$. The time variation of the stochastic potential is expected to damage the ordered component of the motion and to favour the random aspects. It is thus essential to investigate the dependence of $\mathrm{W}$ transport on the decorrelation time $\tau_{d}$. The dependence of the pinch velocity on the energy of the ions $W$ could be important as a control method. The 
other three parameters describe details of the shape of the turbulence EC (44), which are less important for the pinch mechanism produced by the parallel acceleration.

$K_{*}=\Phi / \rho_{*}$ is the measure of turbulence amplitude $\Phi=e A_{\phi} / T_{i}$. The latter is also contained in the parameter of the parallel acceleration $P_{a}$. For a more clear presentation of the results, we analyze the dependence of the $\mathrm{W}$ ion transport on the physical parameters $\Phi$ and $Z$ rather than on $K_{*}$ and $P_{a}$.

The physical range of the parameters is explored around a basic case with $\Phi=0.03, Z=40$ (corresponding to $K_{*}=15, P_{a}=0.09$ ), $\lambda_{z}=0.5, V_{d}=1, \tau_{d}=\infty$ (static potential), $W=1$, $\lambda_{x}=5, \lambda_{y}=2$, and $k_{0}=1$. The units in the figures are $\rho_{i} V_{*}$ for the diffusion coefficient, $\rho_{i}$ for the average trajectories, $V_{*}$ for the pinch velocity and $\tau_{0}$ for the time.

The analysis is performed using numerical simulations (the DNS methods and codes described in Section 3.1).
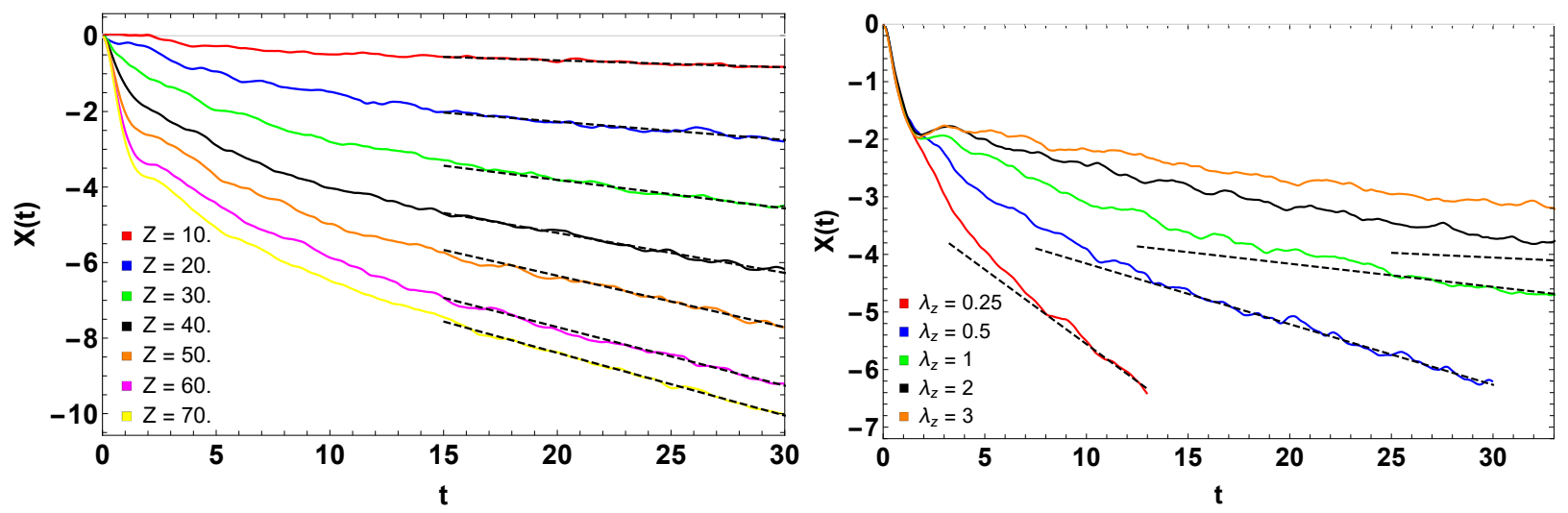

Figure 5: The average radial dispacement of the $\mathrm{W}$ ions for several values of $Z$ (left panel) and of $\lambda_{z}$ (right panel). The other parameters correspond to the basic case.

The existence of the radial pinch velocity can be clearly seen in Fig. 5, which presents examples of radial displacements $X(t)=\langle x(t)\rangle$. A fast increase of $X(t)$ appears in all cases at small times, followed by a transitory evolution (that depends on the parameters of the process), which eventually leads to the asymptotic regime. The latter is always linear in time and corresponds to the (asymptotic) radial pinch. We note that the poloidal average velocity is not invariant as in two-dimensional potentials. Starting from $V_{p}$, it has a transitory variation that can end with a stabilized asymptotic slightly different of $V_{p}$. Examples of the paths of the average trajectories are presented in Fig. 6. They show that the average poloidal motion is not simply $Y(t)=V_{p} t$, but it depends on the other parameters (especially on $\lambda_{z}$, which controls the parallel acceleration).

We present below the results obtained for the dependence of the asymptotic values $V_{x}^{\infty}$ and $D_{x}^{\infty}$ on each parameter. Scaling laws are derived and physical explanations are deduced. The latter are based on the two interaction mechanisms between the parallel motion and the perpendicular transport: the symmetry breaking of the HDs that generates the pinch and 

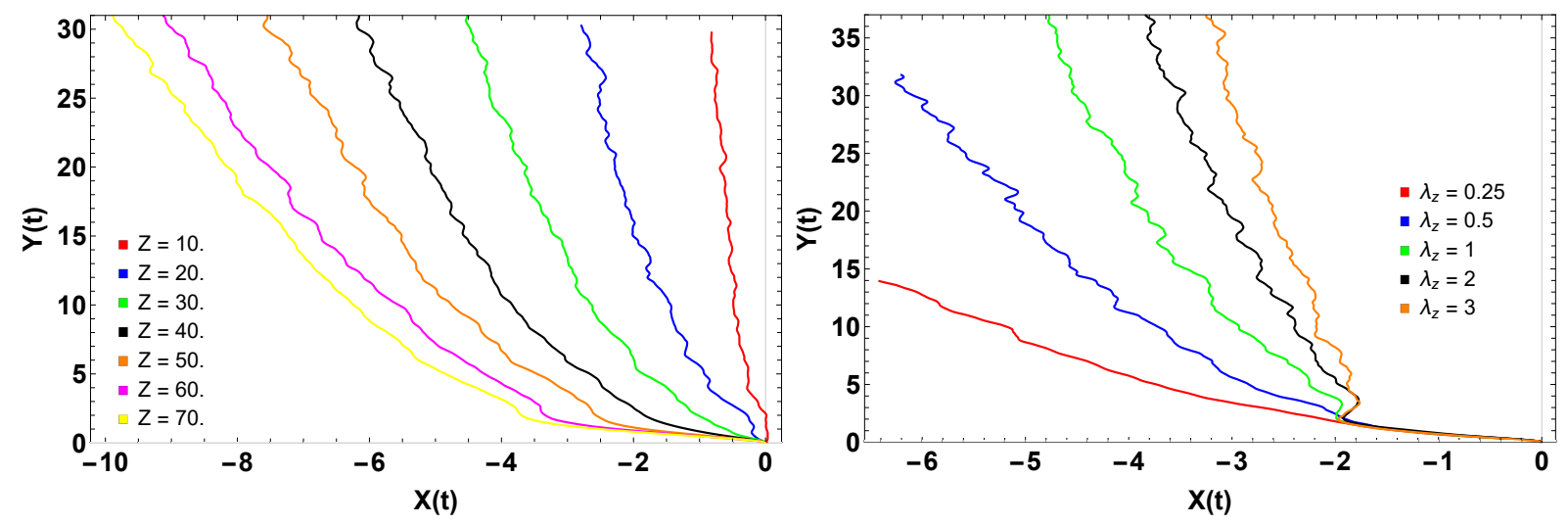

Figure 6: The average paths of the $\mathrm{W}$ ions for several values of $Z$ (left panel) and of $\lambda_{z}$ (right panel). The other parameters correspond to the basic case.

the parallel decorrelation that influences both $V_{x}^{\infty}$ and $D_{x}^{\infty}$. The parallel decorrelation time $\tau_{z}^{\infty}\left(W, Z, \Phi, \lambda_{z}\right)$, Eq. (23), is a decreasing function of $W, \Phi$ and $Z$ and an increasing function of $\lambda_{z}$, as discussed in Section 4 .

- Turbulence amplitude $\Phi$

The amplitude of the turbulence influences the electric drift velocity, the parallel acceleration and the potential energy. It has a complex effect on the pinch velocity and on the diffusion coefficient.

The increase of the electric drift determines the decay of the time of flight as $\Phi^{-1}$, and a stronger transient growth in the quasilinear regime $\left(t<\tau_{f l}\right)$ for both $D_{x}(t) \sim \Phi^{2} t^{2}$ and $V_{x}(t) \sim \Phi t$. Trajectory eddying combined with the increase of the potential energy and of the parallel acceleration modifies the dependence on $\Phi$ in the nonlinear regime, and, consequently, in the asymptotic $V_{x}^{\infty}$ and $D_{x}^{\infty}$.

The asymptotic radial velocity $V_{x}^{\infty}$ is shown in Fig. 7 (left panel) as function of $\Phi$. The pinch is negative (inward) for the whole range of $\Phi$ and it increases with $\Phi$. The dependence is approximately linear for $\Phi \leq 0.04$, and a tendency of saturation can be observed at larger $\Phi$.

The saturation of $V_{x}^{\infty}$ at large $\Phi$ is determined by the energy conservation, which prevents the trajectories to reach the regions with positive values of $\phi$ above the limit $\phi_{\max }$ defined in Eq. (22). This determines the cut of $\langle x(t)\rangle_{\phi^{0}}$ seen in Fig. 2 (right panel), which destroys the equilibrium of the HDs. The maximum perturbation of the HDs corresponds to the limit $Z \Phi \rightarrow \infty$, which eliminates the whole positive range of $\phi^{0}\left(\phi_{\max } \rightarrow 0\right)$. The pinch velocity saturates for $Z \Phi \rightarrow \infty$ (practically for $Z \Phi \gg W$ ) at a value that equals the negative HD.

The asymptotic diffusion coefficient $D_{x}^{\infty}$ increases with the increase of $\Phi$ according to the law $D_{x}^{\infty} \sim \Phi^{\gamma}$ with $\gamma=1.5$, as seen in Fig. 7 (right panel). The values $1<\gamma<2$ define the super-Bohm regime. Such regime is unusual in the presence of trajectory trapping or eddying, which yields the scaling (24) with $0<\gamma<1$. This stronger increase of $D_{x}^{\infty}$ is the effect of the parallel acceleration through the effective decorrelation time $\tau_{z}^{\infty}$. As discussed in Section $4, \tau_{z}^{\infty}$ is a decreasing function of $\Phi$. The supplementary dependence on $\Phi$ through $\tau_{z}^{\infty}(\Phi)$ increases 
the exponent $\gamma$. Thus, the super-Bohm regime is the result of trajectory trapping coupled to the parallel accelerated motion.
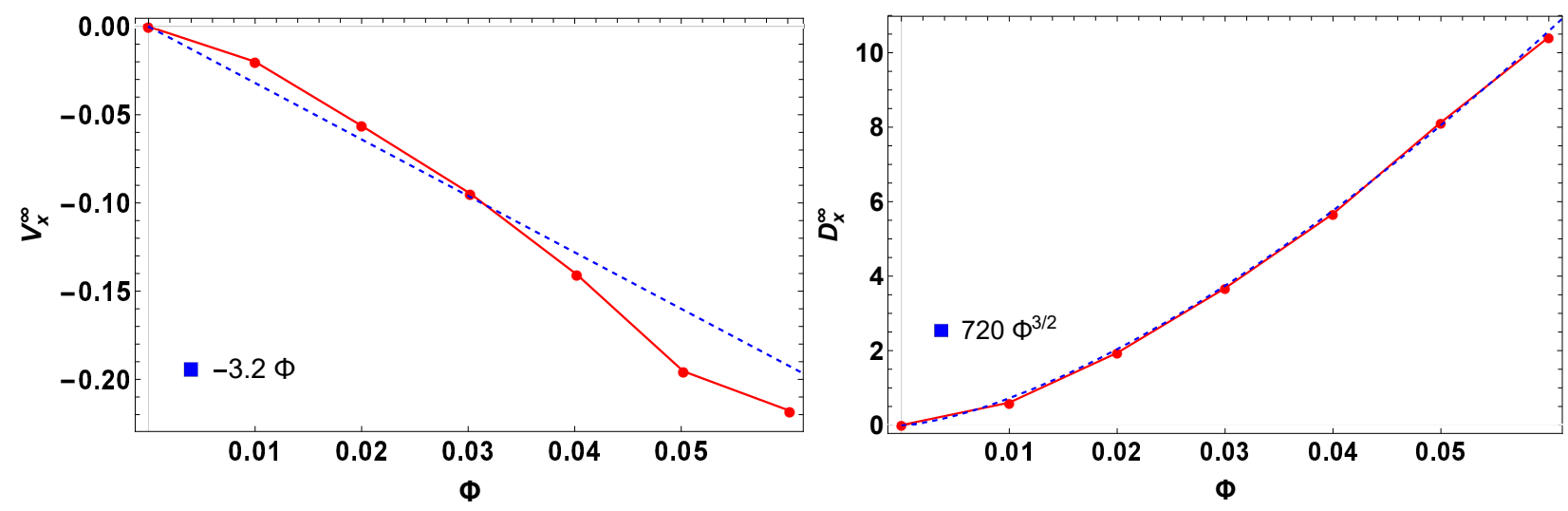

Figure 7: W transport dependence on turbulence amplitude : the asymptotic pinch velocity (left panel) and the diffusion coefficient (right panel).

\section{- Ionization rate $Z$}

The mechanism of generation of the radial pinch depends essentially on the product $Z \Phi$. Thus, the ionization rate has a similar effect with the amplitude $\Phi$ of the turbulence. As seen in Fig. 8 (left panel), the pinch velocity has an approximately linear increase followed by the tendency of saturation, a behaviour that is similar to the dependence on $\Phi$ (Fig. 7 (left panel)). The diffusion coefficient shown in 8 (right panel) has a more complicated dependence on $Z$, but the variation of $D_{x}^{\infty}$ on the relevant range of $Z$ is small (of the order $\pm 20 \%$ of the average). The influence of $Z$ on the diffusion is produced through the effective parallel decorrelation time $\tau_{z}^{\infty}$ that depends on $Z$.
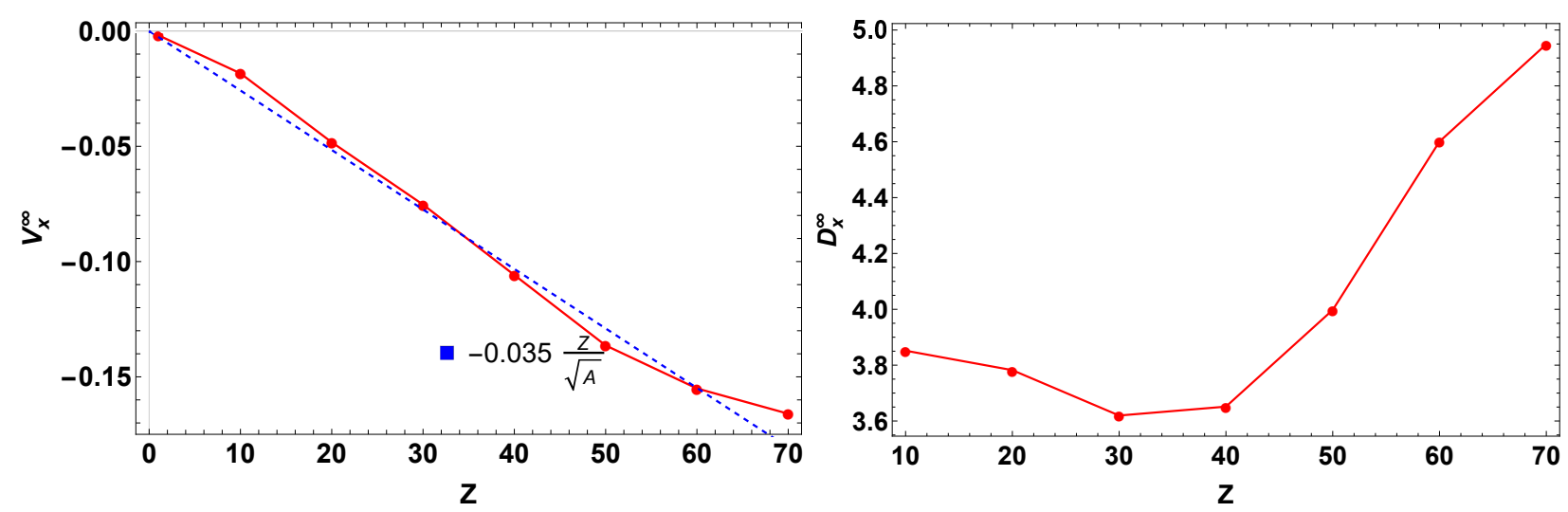

Figure 8: W transport dependence on the ionization rate $Z$ : the asymptotic pinch velocity (left panel) and the diffusion coefficient (right panel). 

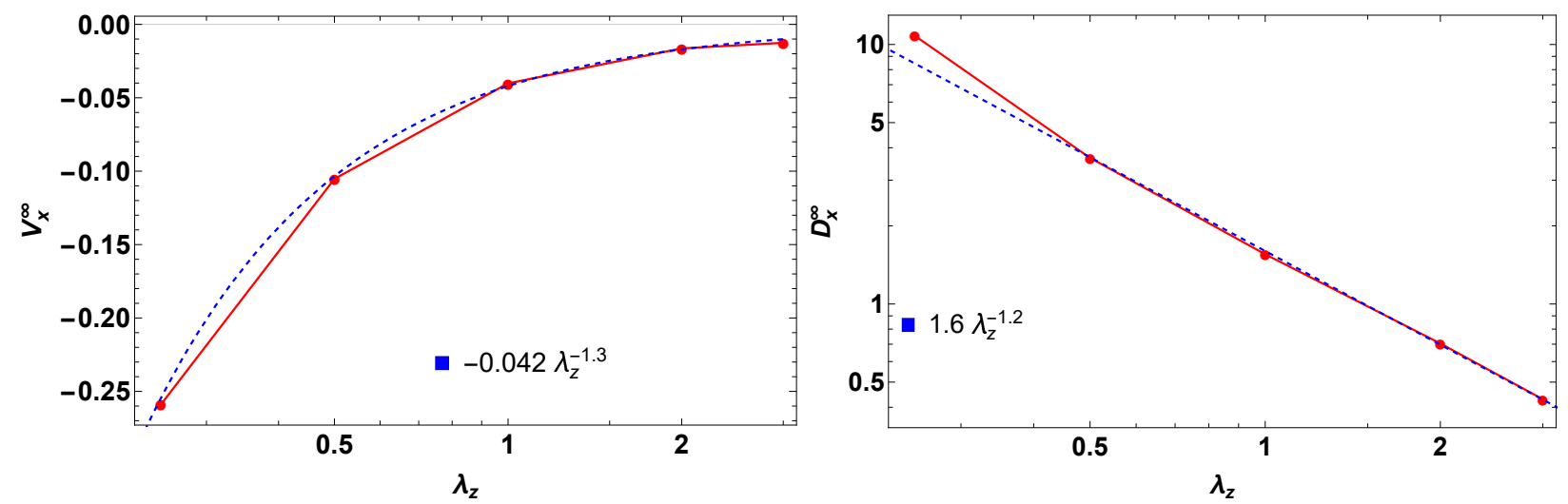

Figure 9: W transport dependence on the parallel correlation length $\lambda_{z}$ : the asymptotic pinch velocity (left panel) and the diffusion coefficient (right panel).

- Parallel correlation length $\lambda_{z}$

The pinch mechanism analyzed here appears only in three-dimensional stochastic potentials. But, as discussed in Section $5, V_{x}^{\infty}$ essentially results from the symmetry breaking of the HDs determined by the energy conservation. The potential energy does not dependent on $\lambda_{z}$, which means that a finite $\lambda_{z}$ is necessary, but its direct quantitative influence on the pinch mechanism is small.

However, $\lambda_{z}$ has a strong influence on the transport through the parallel decorrelation time $\tau_{z}^{\infty}$ in Eq. (23) that increases with $\lambda_{z}$ faster than linearly. It explains the large decrease rate of both $V_{x}^{\infty}$ and $D_{x}^{\infty}$ seen in Fig. 8, which shows that $\left|V_{x}^{\infty}\right| \sim \lambda_{z}^{-1.3}$ and $D_{x}^{\infty} \sim \lambda_{z}^{-1.2}$.

Thus, $\lambda_{z}$ determines the decrease of $V_{x}^{\infty}$ and $D_{x}^{\infty}$ only through the modification of the $\tau_{z}^{\infty}$.

- Poloidal velocity $V_{p}$

The poloidal average velocity is the source of the hidden drifts. It has a strong influence on both the pinch velocity and the diffusion coefficient, as seen in Fig. 10.

The equations of motion (11)-(2) are invariant at the change $V_{p} \rightarrow-V_{p}$ and $\mathbf{x} \rightarrow-\mathbf{x}$, which implies that the conditional displacements and the HDs change their sign when $V_{p} \rightarrow-V_{p}$. Thus, the pinch velocity $V_{x}^{\infty}$ is an anti-symmetrical function of $V_{p}$, as seen in Fig. 10 (left panel). $V_{x}^{\infty}$ is linear in $V_{p}$ at small $V_{p}$, it has a maximum at $V_{p} \cong 0.3$ and a long tail with $V_{x}^{\infty} \sim V_{p}^{-1.2}$ at large $V_{p}$.

The direction of the pinch produced by the parallel acceleration can be changed from inward to outward by inversing the orientation of the poloidal velocity.

The diffusion coefficient is strongly influenced by $V_{p}$, which determines a large decrease of $D_{x}^{\infty}$, as seen in Fig. 10 (right panel).

Thus, $V_{p}$ has a special effect on the transport, different compared to the other parameters. The pinch velocity $V_{x}^{\infty}$ is modified because $V_{p}$ influences the amplitude of the HDs. The diffusion coefficient $D_{x}^{\infty}$ is modified because $V_{p}$ influences the structure of the contour lines of the total potential. 

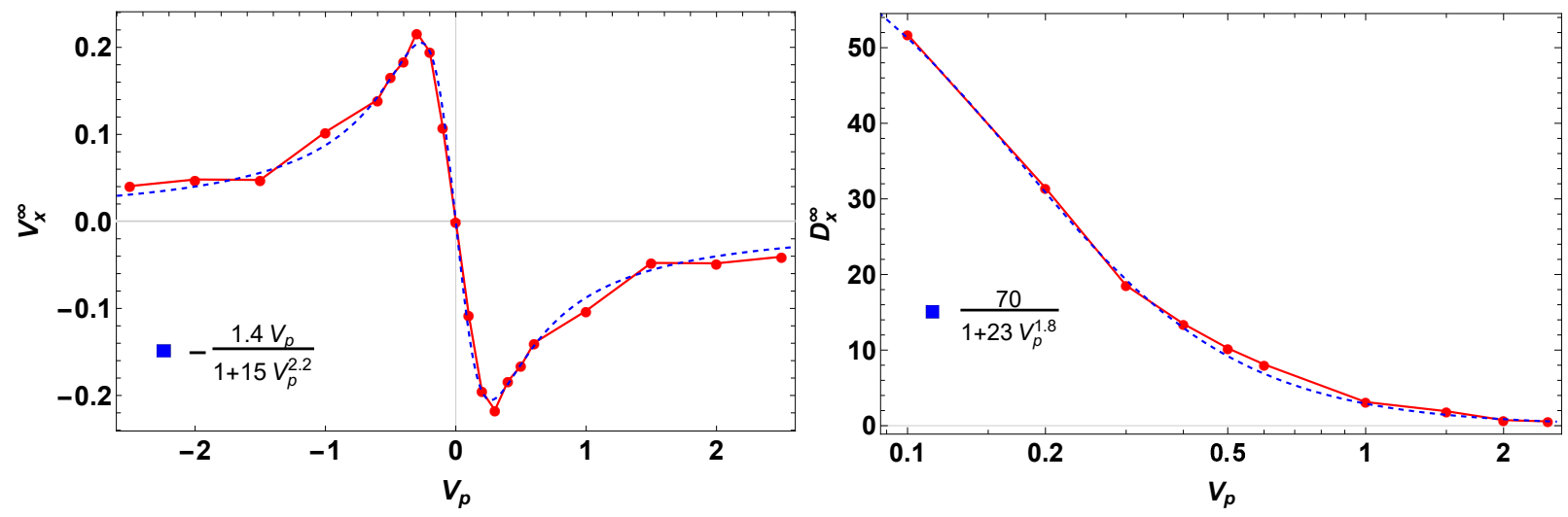

Figure 10: W transport dependence on the poloidal velocity $V_{p}$ : the asymptotic pinch velocity (left panel) and the diffusion coefficient (right panel).

\section{- Time decorrelation $\tau_{d}$}

Our results have confirmed the idea that the time variation of the stochastic potential determines a process of elimination of the pinch velocity by strengthening the random aspects of the motion. In addition to this, the Lagrangian energy is not a constant, but a fluctuating function of time.

However, the pinch velocity survives in time dependent potentials $\phi(\mathbf{x}, z, t)$ if the decorrelation tine $\tau_{d}$ is not too small. As seen in Fig. 11 (left panel), $V_{x}^{\infty}$ is weakly dependent on $\tau_{d}$ for $\tau_{d}>1$, and it has fast decrease as $V_{x}^{\infty} \sim-\tau_{d}^{2}$ for $\tau_{d}<0.5$. The pinch velocity is eliminated for fast time variation with $\tau_{d} \ll \tau_{f l}$.

A different behaviour was obtained for $D_{x}^{\infty}$. As seen in Fig. 11 (right panel), $D_{x}^{\infty}$ increases at small $\tau_{d}$ (in the quasi-linear regime, $\tau_{d} \ll \tau_{f l}$ ), reaches a maximum and decreases due to trapping for $\tau_{d} \gg \tau_{f l}$. At larger $\tau_{d}$, of the order of the parallel decorrelation time, $D_{x}^{\infty}$ saturates at the value corresponding to the static potential. This behaviour results from the combination of the time decorrelation processes produced by the time variation of the potential (represented by the time dependence of the EC (5)) and by the parallel motion (19). This yields an effective decorrelation time $\tau^{\infty}$ that is $\tau^{\infty} \cong \tau_{d}$ for $\tau_{d} \ll \tau_{z}^{\infty}$ and $\tau^{\infty} \cong \tau_{z}^{\infty}$ for $\tau_{d} \gtrsim \tau_{z}^{\infty}$.

- Energy of the W ions

The energy is directly connected to the mechanism of pinch generation. The cut of the conditional average displacements $\langle\mathbf{x}(t)\rangle_{\phi^{0}}$, which has the dominant influence of the asymmetry of the HDs, appears at $\phi_{\max }=W /(Z \Phi)$. It is expected that the change of $W$ determines a variation of the pinch velocity of the order of those produced by $Z$ or $\Phi$, but inverse, in the sense that $\left|V_{x}^{\infty}\right|$ is a decreasing function of $W$.

We have obtained only a very weak decrease of the pinch velocity with the energy.

The reason is the dependence of the parallel decorrelation time $\tau_{z}^{\infty}$, which is a decreasing function of $W$. The saturation of $V_{x}(t)$ at a smaller time determines the increase of $\left|V_{x}^{\infty}\right|$ (because it is a decreasing function of $t$ ). The two effects of the parallel acceleration (symmetry breaking 

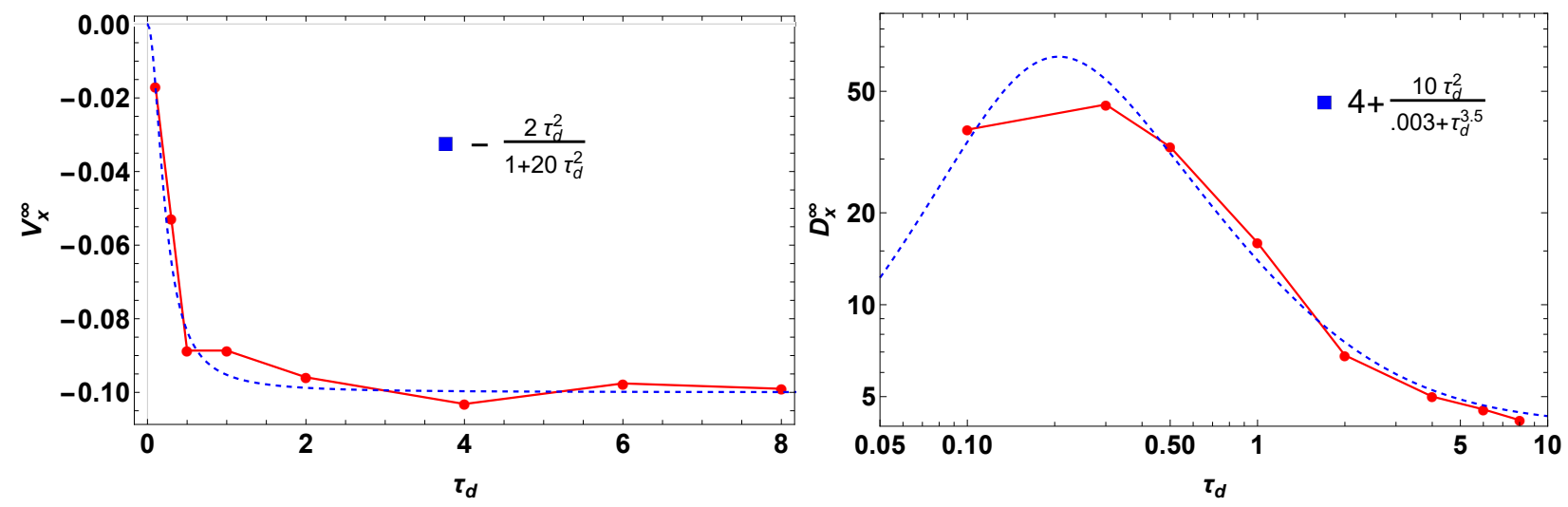

Figure 11: $\mathrm{W}$ transport dependence on the time variation parameter $\tau_{d}$ : the asymptotic pinch velocity (left panel) and the diffusion coefficient (right panel).

of the HDs and decorrelation) are opposite in this case of the energy dependence, and they partly compensate.

The increase of the energy also determines a weak increase of the diffusion coefficient.

\section{Discussions and conclusions}

The main finding of this work is a radial pinch that is generated by the stochastic parallel acceleration in turbulent plasmas. It is significant for high $Z$ impurities and negligible for plasma ions. We have shown that the pinch is produced in three-dimensional turbulence by the interaction of the parallel motion with the HDs, a special type of quasi-coherent radial motion that appears due to a poloidal average velocity.

We have also shown that the influence of the parallel motion on the transport through the parallel decorrelation time $\tau_{z}^{\infty}$ is much stronger for heavy impurities than for plasma ions. The fluctuations of the parallel velocity are very large for $\mathrm{W}$ ions, and they determine a smaller parallel decorrelation time that depends on the parameters of the parallel motion $\tau_{z}^{\infty}\left(W, Z, \Phi, \lambda_{z}\right)$. This complex decorrelation process influences both the pinch velocity and the diffusion coefficient. It leads to an unusual diffusion regime of super-Bohm type and modifies the scaling laws of $V_{x}^{\infty}$ and $D_{x}^{\infty}$.

The physical domains of the main parameters of the transport model were explored for evaluating the scaling laws and for obtaining the range of the normalized pinch velocity and diffusion coefficient. The typical values of $\left|V_{x}^{\infty}\right|$ are in the interval $(0.05,0.25)$.

We underline that the dependence of $V_{x}^{\infty}$ on $V_{p}$ (Fig. 10 (left panel)) provides a very efficient control possibility. The change of $\mathbf{V}_{p}$ from the direction of the electron to the ion diamagnetic velocity determines the inversion of the pinch from inward to outward direction. A strong variation of $V_{x}^{\infty}$ with $V_{p}$ exists at small $\left|V_{p}\right| \lesssim 0.5$, which shows a high sensitivity of the pinch velocity to the poloidal velocity.

The relevance of the pinch velocity for ASDEX Upgrade, JET and ITER can be evaluated 
from the dimensionless results. The main difference (concerning $V_{x}^{\infty}$ ) between present plasmas and ITER is the electron temperature. Due to the time-scale separation of the atomic and transport processes, the $\mathrm{W}$ impurities are in coronal equilibrium. The fractional abundance of each ionization stage is a function of the electron temperature that is practically not influenced by the transport [44. This determines different ranges of the ionization rates for the present plasmas and ITER. In the first case $Z$ varies from boundary to the center in the interval $(20,48)$, while in the second case the interval is $(45,63)$. Typical values of $Z$ in the core plasma are $Z=[43,44,57]$, where the first value in this and the following triads corresponds to ASDEX Upgrade, the second to JET and the third to ITER. This determines normalized values of the pinch velocity of the order $\left|V_{x}^{\infty}\right| \approx[0.10,0.11,0.15]$. Using typical parameters of these plasmas, the pinch velocities are of the order $\left|V_{x}^{\infty}\right| \approx[160,110,194] \mathrm{m} / \mathrm{sec}$. Thus, the pinch velocity is larger in the ITER plasmas roughly by $50 \%$ at similar parameters of the turbulence and poloidal velocity. The convection time to plasma center is very small $\Delta t_{c}=a / V_{x}^{\infty} \approx[4,11,10] \mathrm{msec}$. Convection dominates diffusion in all cases, because $\Delta t_{c} \ll \Delta t_{\text {dif }}$, where $\Delta t_{\text {dif }}=a^{2} /\left(2 D_{x}^{\infty}\right)$ is the diffusive time. The ratio $r=\Delta t_{c} / \Delta t_{\text {dif }}$ is $r \approx[0.18,0.10,0.07]$.

The above estimation shows a very strong effect of the acceleration induced pinch on the dynamics of the $\mathrm{W}$ impurities. This enables the idea that the processes found here remain significant in a frame of a realistic transport model that includes $\mathrm{W}$ ion collisions, the polarization drift and neoclassical aspects. The future work will be dedicated to the development of the model and to the examination of the of interaction of $V_{x}^{\infty}$ with other types of radial pinches.

In conclusion, this study provides understanding of the complex processes of interaction of the parallel acceleration with the perpendicular transport. The main effect consists of the generation of a radial pinch that appears to be significant for the dynamics of the $\mathrm{W}$ impurities in present plasmas and ITER.

Acknowledgements This work has been carried out within the framework of the EUROfusion Consortium and has received funding from the Euratom research and training programme 20142018 and 2019-2020 under grant agreement No 633053 and from the Romanian Ministry of Research and Innovation. The views and opinions expressed herein do not necessarily reflect those of the European Commission.

\section{References}

[1] Philipps V. 2011 Tungsten as material for plasma-facing components in fusion devices $J$. Nucl. Mater. 415 S2R

[2] Neu, V. Bobkov, R. Dux, A. Kallenbach, T. Pütterich, H. Greuner, O. Gruber, A. Herrmann, C. Hopf, K. Krieger et al., J. Nucl. Mater. 363-365, 52 (2007).

[3] R. Dux, R. Neu, A. G. Peeters, G. Pereverzev, A. Muck, F. Ryter, J. Stober and ASDEX Upgrade Team, Plasma Phys. Controlled Fusion 45, 1815 (2003).

[4] Bock A. et al (ASDEX Upgrade Team) 2018 Advanced tokamak investigations in fulltungsten ASDEX Upgrade Phys. Plasmas 25056115 
[5] Pütterich T. et al (The ASDEX Upgrade Team and JET EFDA Contributors) 2013 Observations on the $\mathrm{W}$-transport in the core plasma of JET and ASDEX Upgrade Plasma Phys. Control. Fusion 55124036

[6] Puiatti M. E. et al (JET-EFDA Contributors) 2006 Phys. Plasmas 13042501

[7] Valisa M. et al (JET-EFDA Contributors) 2011 Nucl. Fusion 51, 033002

[8] Bucalossi J. et al 2014 The WEST project: Testing ITER divertor high heat flux component technology in a steadystate tokamak environment Fusion Eng. Des. 89907

[9] Krupin V.A. et al 2017 Experimental study of tungsten transport properties in T-10 plasma Nucl. Fusion 57066041

[10] Nakano T. et al 2009 Tungsten accumulation in H-mode plasmas of JT-60U Nucl. Fusion 49115024

[11] Sertoli M., Angioni C. and Odstrcil T. (ASDEX Upgrade Team, and EUROFusion MST1 Team) 2017 Parametric dependencies of the experimental tungsten transport coefficients in ICRH and ECRH assisted ASDEX Upgrade H-modes Phys. Plasmas 24, 112503

[12] Hogeweij G.M.D. et al () 2015 ITER-like current ramps in JET with ILW: experiments, modelling and consequences for ITER Nucl. Fusion 55013009

[13] Espinosa S. and Catto P. J. 2017 Pedestal radial flux measuring method to prevent impurity accumulation Phys. Plasmas 24, 055904

[14] Raghunathan M. et al () 2017 Heavy impurity confinement in hybrid operation scenario plasmas with a rotating 1/1 continuous mode Plasma Phys. Control. Fusion 59124002

[15] Köchl F. et al () $2018 \mathrm{~W}$ transport and accumulation control in the termination phase of JET H-mode discharges and implications for ITER Plasma Phys. Control. Fusion 60 074008

[16] Angioni C. and Helander P. 2014 Neoclassical transport of heavy impurities with poloidally asymmetric density distribution in tokamaks Plasma Phys. Control. Fusion 56124001

[17] Casson F. J. (JET EFDA Contributors, and ASDEX-Upgrade Team) 2014 Theoretical description of heavy impurity transport and its application to the modelling of tungsten in JET and ASDEX Upgrade Plasma Physics and Controlled Fusion 57, 1-9

[18] Moradi S., Fülöp T., Mollén A. and Pusztai I. 2011 A possible mechanism responsible for generating impurity outward flow under radio frequency heating Plasma Phys. Control. Fusion 53, 115008

[19] Garbet X. et al 2005 Turbulent fluxes and entropy production rate Phys. Plasmas 12, 082511

[20] Breton S. et al (JET Contributors) 2018 High Z neoclassical transport: Application and limitation of analytical formulae for modelling JET experimental parameters Phys. Plasmas 25012303

[21] F. J. Casson, C. Angioni, E. A. Belli, R. Bilato, P. Mantica, T. Odstrcil, T. Pütterich, M. Valisa, L. Garzotti, C. Giroud, J. Hobirk, C. F. Maggi, J. Mlynar, and M. L. Reinke, Plasma Phys. Control. Fusion 57, 014031 (2015). 
[22] Manas P. et al (JET contributors) 2017 Gyrokinetic modeling of impurity peaking in JET H-mode plasmas Phys. Plasmas 24062511

[23] Guirlet R. et al 2010 Particle transport in low core turbulence Tore-Supra plasmas Nucl. Fusion 50, 095009

[24] Nicolas T., Lütjens H., Luciani J.-F., Garbet X. and Sabot R. 2014 Impurity behavior during sawtooth activity in tokamak plasmas Phys. Plasmas 21012507

[25] Breton S. et al 2018 First principle integrated modeling of multi-channel transport including Tungsten in JET Nucl. Fusion 58096003

[26] Koechl F. et al () 2017 Modelling of transitions between L- and H-mode in JET high plasma current plasmas and application to ITER scenarios including tungsten behaviour Nucl. Fusion 57086023

[27] Yamoto S., Bonnin X., Homma Y., Inoue H., Hoshino K., Hatayama A. and Pitts R.A. 2017 Kinetic modeling of high-Z tungsten impurity transport in ITER plasmas using the IMPGYRO code in the trace impurity limit Nucl. Fusion 57116051

[28] Casson F.J. et al (JET EFDA Contributors8 and ASDEX-Upgrade Team) 2020 Theoretical description of heavy impurity transport and its application to the modelling of tungsten in JET and ASDEX upgrade Nucl. Fusion 60066029

[29] Linder O. et al (the EUROfusion MST1 Team) 2019 Flux-driven integrated modelling of main ion pressure and trace tungsten transport in ASDEX Upgrade Nucl. Fusion 59 016003

[30] Ferrari H. E., Farengo R. and Clauser C. F. 2019 The effect of a saturated kink on the dynamics of tungsten impurities in the plasma core Plasma Phys. Control. Fusion 61 035010

[31] Vlad M. and Spineanu F. 2018 Hidden drifts in turbulence Europhysics Letters (EPL) 124, 60002

[32] Palade D. I. and Vlad M. 2020 Fast generation of Gaussian random fields for direct numerical simulations of stochastic transport Electronic preprint arXiv:2006.11106 [physics.comp$\mathrm{ph}]$

[33] Vlad M., Spineanu F., Misguich J. H. and Balescu R. 1998 Diffusion with intrinsic trapping in 2-d incompressible velocity fields Phys. Rev. E 587359

[34] Vlad M. and Spineanu F. 2013 Test particle study of ion transport in drift type turbulence Phys. Plasmas 20122304

[35] Vlad M. and Spineanu F. 2015 Electron heat transport multi-scale turbulence Phys. Plasmas 22112305

[36] Vlad M. and Spineanu F. 2016 Direct effects of the resonant magnetic perturbation on turbulent transport Nuclear Fusion 56092003

[37] Vlad M. and Spineanu F. 2017 Randon and quasi-coherent aspects in particle motion and their effects on transport and turbulence evolution New Journal of Physics 19025014 
[38] Vlad M., Spineanu F., Misguich J. H. and Balescu R. 2002 Electrostatic turbulence with finite parallel correlation length and radial diffusion Nucl. Fusion 42157

[39] Vlad M., Spineanu F. and Benkadda S. 2006 Impurity pinch from a ratchet process Phys. Rev. Lett. 96085001

[40] Spizzo G., White R. B. and Cappello S. 2007 Chaos generated pinch effect in toroidal confinement devices Phys. Plasmas 14102310

[41] Vlad M. 2018 Effects of the mean field gradients on magnetic field line random walk Astrophysical J. 867:104

[42] Vlad M. and Spineanu F. 2018 Combined effects of hidden and polarization drifts on impurity transport in tokamak plasmas Phys. Plasmas 25092304

[43] Hauff T and Jenko F 2007 Phys. Plasmas 14092301

[44] Putterich T., Neu R., Dux R., Whiteford A. D. and O'Mullane M. G. 2008 Modelling of measured tungsten spectra from ASDEX Upgrade and predictions for ITER Plasma Phys. Control. Fusion 50085016 


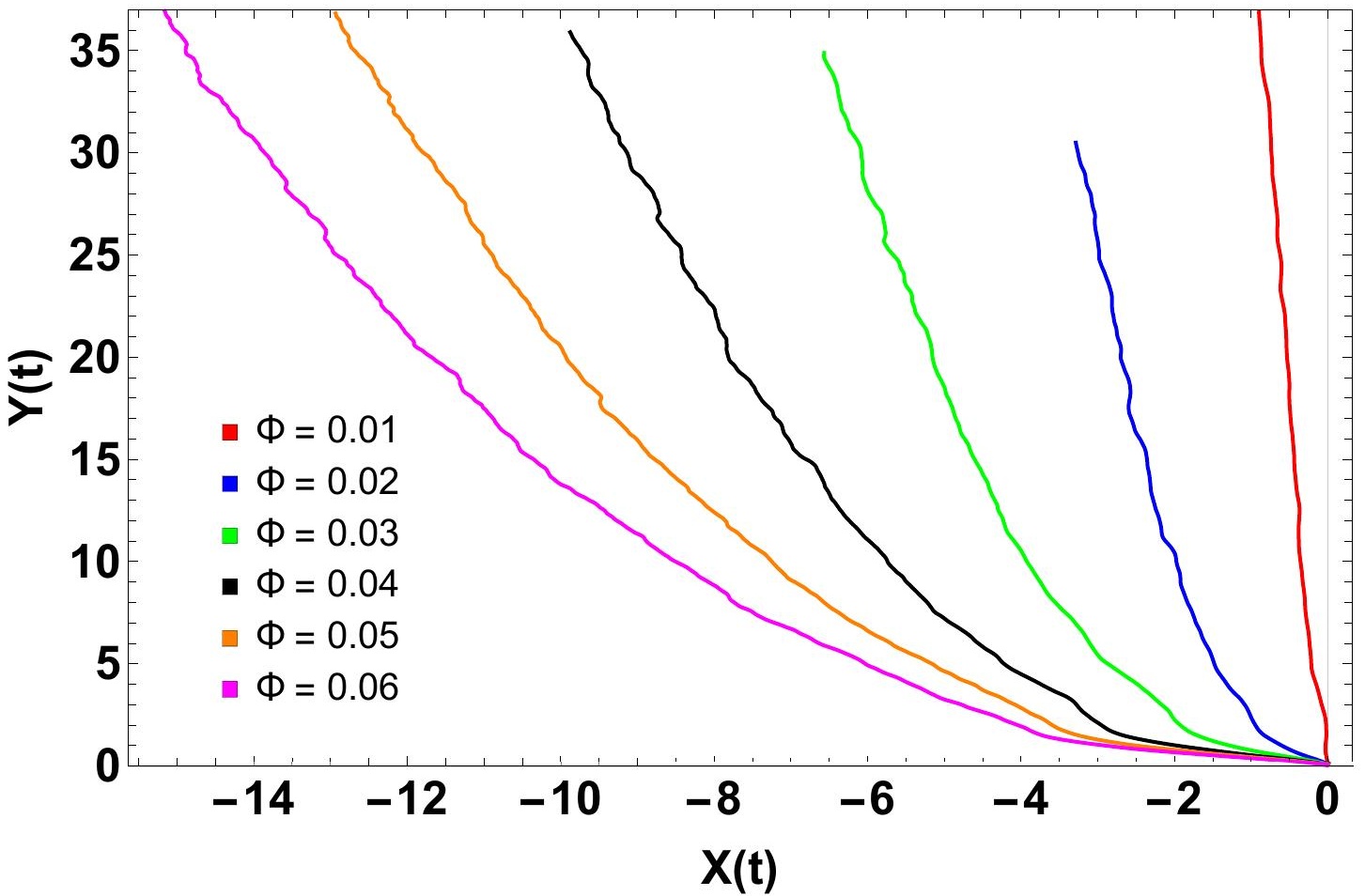




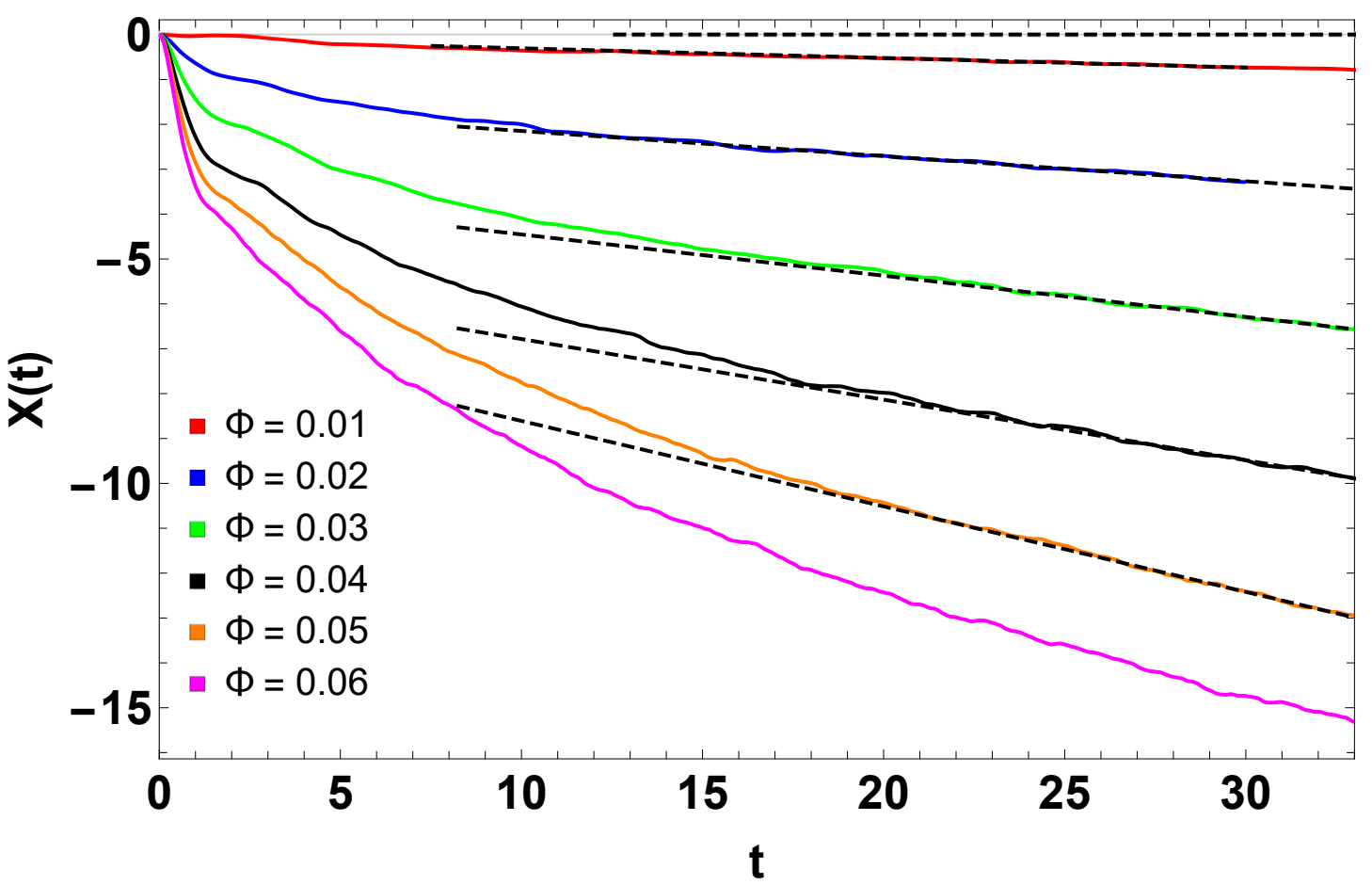




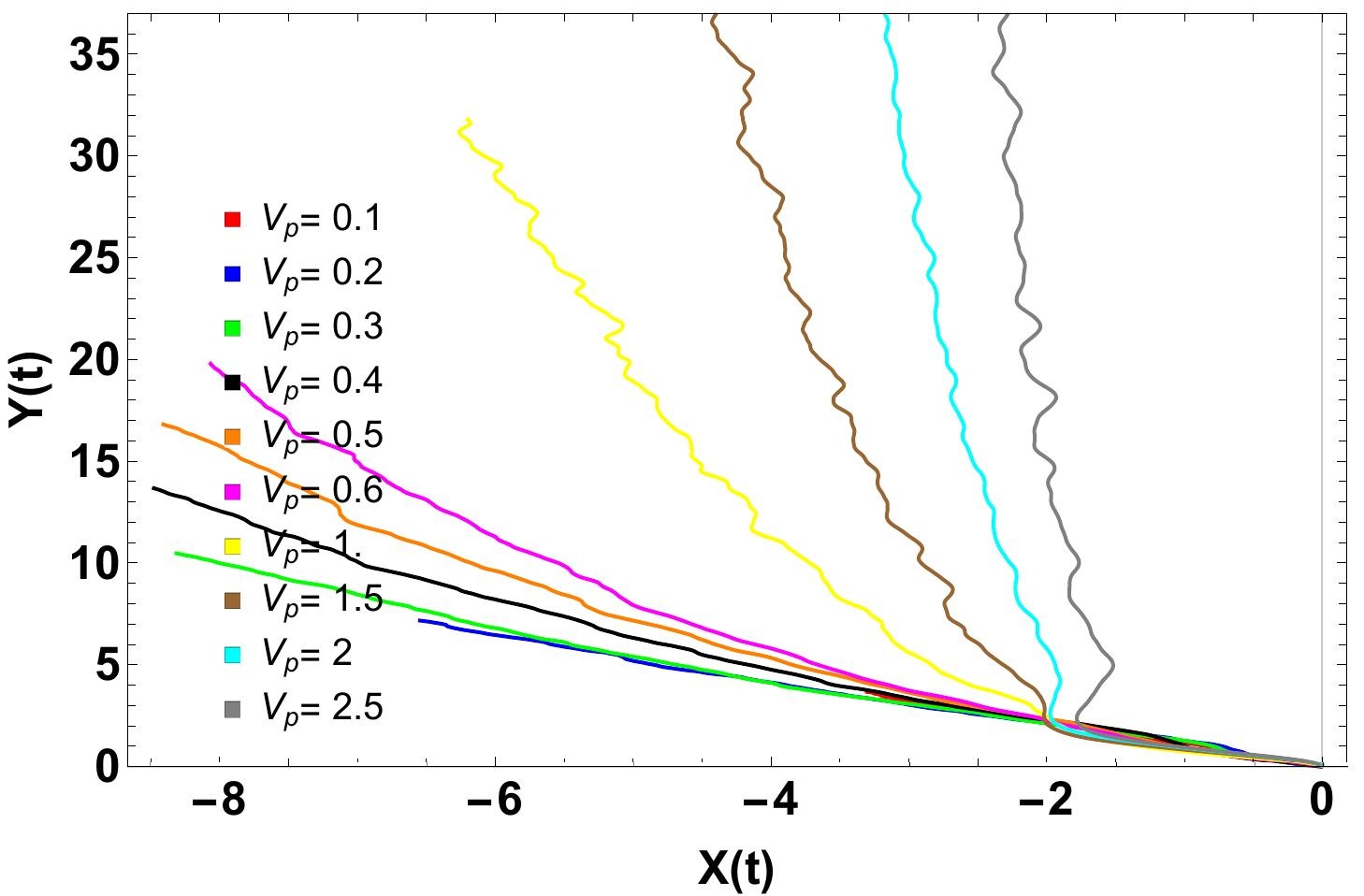




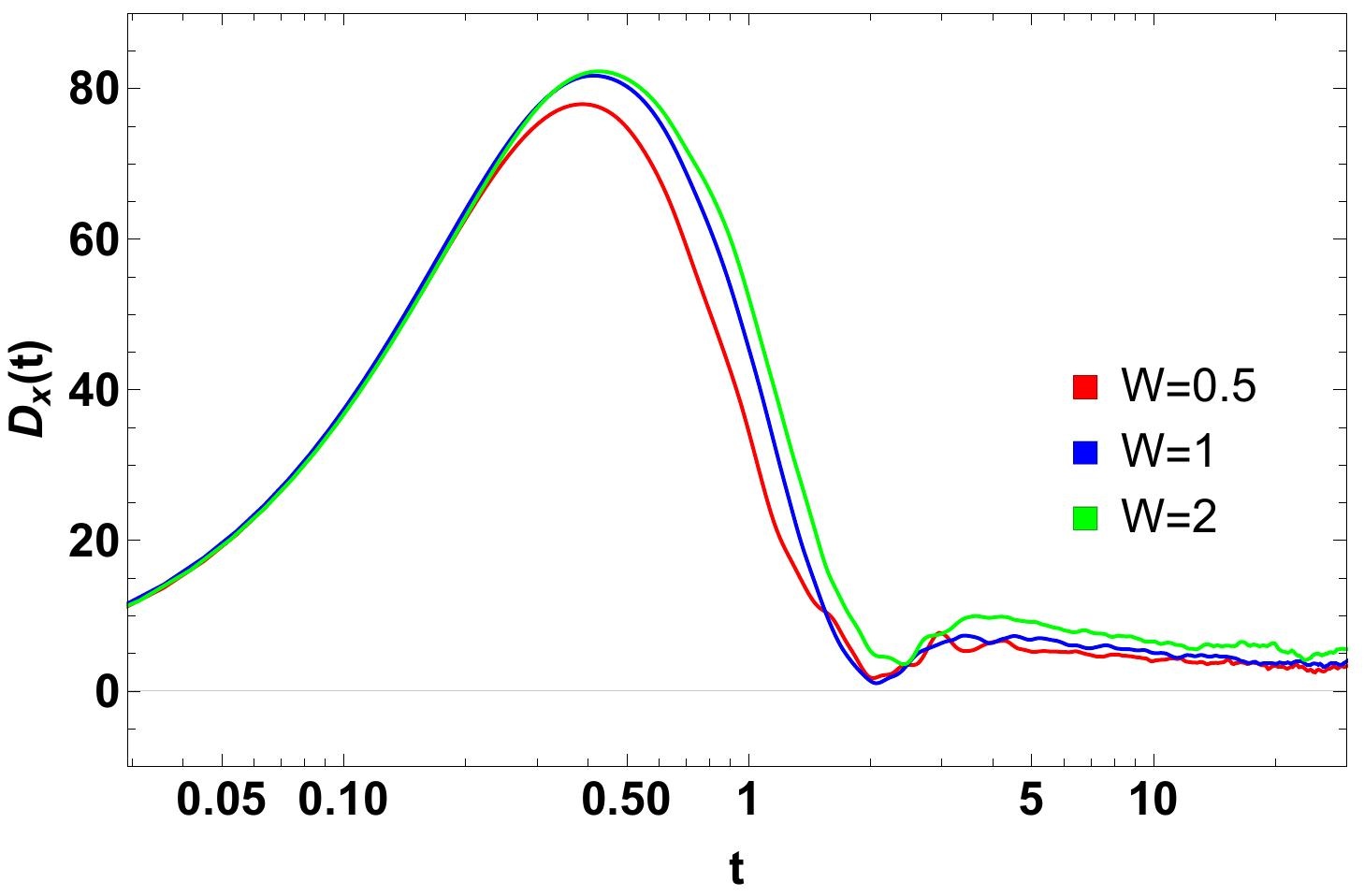




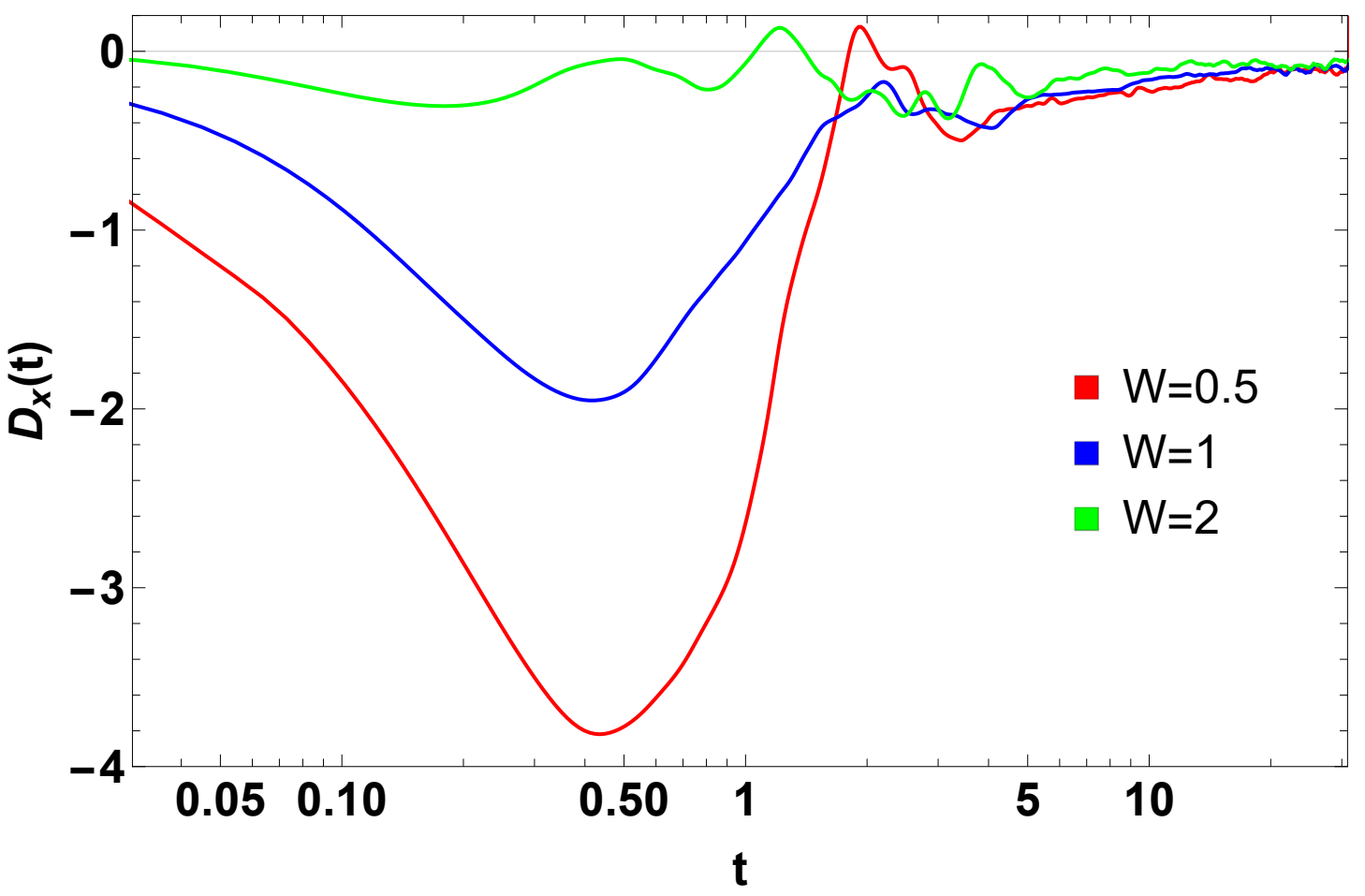

